\title{
Development of a Discrete Element Model with Moving Realistic Geometry to Simulate Particle Motion in a Mi-Pro Granulator
}

\author{
N. J. Watson ${ }^{1 *}$, M. J. W. Povey ${ }^{2}$, G.K. Reynolds ${ }^{3}$, Y. Ding ${ }^{4}$, B. H. Xu ${ }^{5}$ \\ ${ }^{1}$ Department of Chemical and Environmental Engineering, University of Nottingham, University \\ Park, Nottingham NG7 2RD, UK. \\ ${ }^{2}$ School of Food Science and Nutrition, University of Leeds, Woodhouse lane, Leeds LS2 9JT, UK \\ ${ }^{3}$ Pharmaceutical development, AstraZeneca, Silk Road Business Park, Macclesfield, SK10 2NA, UK \\ ${ }^{4}$ College of Engineering and Physical Sciences, University of Birmingham, Edgbaston, Birmingham, \\ B15 2TT, UK \\ ${ }^{5}$ Institute of Particle Science and Engineering, University of Leeds, Woodhouse lane, Leeds LS2 9JT, \\ UK. \\ *Corresponding Author. \\ Address: Dr Nicholas James Watson. Room B16, Coates Building, University Park, University of \\ Nottingham, Nottingham, Nottinghamshire, NG7 2RD, UK. \\ Email: nicholas.watson@ nottingham.ac.uk \\ Phone: (+44) 1157484848
}

Keywords: discrete element method, High shear mixers, particle velocity field, contact detection, moving boundary

\begin{abstract}
This paper presents the implementation of a methodology incorporating a 3D CAD geometry into a 3D Discrete Element Method (DEM) code; discussing some of the issues which were experienced. The 3D CAD model was discretised into a finite element mesh and the finite wall method was employed for contact detection between the elements and the spherical particles. The geometry was based on a lab scale Mi-Pro granulator. Simulations were performed to represent dry particle motion in this piece of equipment. The model was validated by high speed photography of the particle motion at the surface of the Mi-Pro's clear bowl walls. The results indicated that the particle motion was dominated by the high speed impeller and that a roping regime exists. The results from this work give a greater insight into the particle motion and can be used to understand the complex interactions which occur within this equipment.
\end{abstract}

\section{Introduction}

Discrete Element Method (DEM) is a particle modelling technique which allows all the particles within the system to move individually and interact at contact points. The advantage of the technique is that all the data for every particle is accessible at any stage of the simulation. The limitation of the technique is the number of particles which can be modelled or the length of a simulation. This is due to the small time steps and large number of calculations performed. The DEM technique resolves contacts over several time steps. In a given time step it is assumed that disturbances of a particle can propagate no further than its immediate neighbours. If this condition is satisfied then at any given time the resultant force acting on a particle is determined entirely by the interactions with its neighbour particles, which are in contact with it. DEM was initially proposed by Cundall and Strack (1979) for studying soil mechanics. Since then it has been applied to industries such as mining (Cleary, 2000; 
Djordjevic et al., 2004), pharmaceutical (Kwan et al., 2005; Moreno-Atanasio and Ghadiri, 2006), agricultural (Tijskens et al., 2003), food (Raji and Favier, 2004), and chemicals (Kaneko et al., 1999).

DEM has being used extensively to study particle flow in vertical shaft mixers and granulators. The majority of this work has focussed on simple paddle mixers with flat or inclined blades (Stewart et al., 2001; Kuo et al., 2004; Sato et al., 2008; Remy et al., 2010, Remy et al., 2011; Hua et al., 2013).

However, more complex geometries such as the three bladed VG-01 (Terashita et al., 2002) have also being studied. Some of these models were validated using either Particle Emission Positron Tracking (PEPT) (Stewart et al., 2001, Kuo et al., 2004) or high speed photography (Remy et al., 2010). In addition to particle flow the DEM has also being used to investigate mixing in vertical shaft mixers (Sinnot and Cleary, 2003; Zhou et al., 2003; Zhou et al., 2004; Chandratilleke et al., 2012; Radl et al., 2010) and has been used to study certain aspects of the granulation process (Nakamura et al., 2013 and Hassanpour et al., 2013).

A major issue within any discrete element model is how the geometry is represented. Especially as most models are representative of real-life systems, with complex geometries. The more complex the geometry incorporated the more difficult the contact detection process between the particles and the geometry becomes, affecting the simulation time. Three techniques exist to represent complex geometries: Mathematical representation; discrete element mesh and constrained spherical particles. Mathematical representation is the simplest but is only suitable for very basic geometries such as squares and cubes. Representing the geometry as attached spheres makes the contact detection process simpler but does not allow for the detail of the geometry to be recreated accurately and effects the validity of the model. Therefore discrete element meshes are the most accurate and commonly used method for representing geometries.

The purpose of the mesh is to represent a continuum, in this case surface area, with a series of small discrete elements. These elements recreate the continuum and are easier to incorporate numerically into a DEM model. Contact between the discrete triangular elements and the spherical particles can be determined by the finite wall method of Kremmer and Favier (2001a). These authors also described how to incorporate moving parts into DEM models (Kremmer and Favier, 2001b). Although finite element meshes allow for representation of very complex geometries and can handle movement of internal parts, the drawback is computational expense. New algorithms must be included to detect contacts between particles and triangular wall elements, as well as the original particle-particle contacts. Particle-triangle contacts are more complicated to detect, as contact can occur on the surface of the triangle or on one of the edges or vertices. An important issue when constructing finite element meshes is the number of elements used. The more elements used the more accurately the continuum is recreated, but with the cost of an increased simulation time.

If the DEM model requires motion of the geometry, to represent an impeller in a mixer or the belt of a moving conveyor this can be performed using the mesh data. At each time step the nodes at the vertices of the element can be moved from their current location to the new location according to the set motion. Finite element meshes have been used in DEM models of industrial granular flows (Cleary and Sawley, 2002), and high shear mixers (Terashita et al., 2002).

This work will discuss how a 3D geometry was incorporated into an existing DEM code and the techniques developed for contact detection and motion of the impellor. How simulation parameters such as spring stiffness and simulation time step were calculated will also be presented. The code was then validated using high speed photography and used to simulate particle motion in a Mi-Pro lab scale granulator. These granulators are used to investigate granulation at a small scale (Gamble et al., 2009 and Cavinato et al., 2013). They utilise an impeller with three blades inclined backwards at 45 degrees. As this equipment has a nonstandard geometry it is important to understand the particle flows within them. The DEM model developed in this work was then used to investigate the complex particle flow patterns and particle contacts which exist in this equipment. The results from this modelling study can then be used to develop a deeper understanding understand how granulation will occur in these types of devices. 


\section{Discrete Element Method}

The DEM technique uses Newton's second law of motion to calculate the acceleration of a particle due to all of the forces acting on it. Integration of the acceleration twice produces the particle's displacement. A particle can have two types of motion, translational and rotational. The translation motion can be calculated from:

$m_{i} \frac{d \mathbf{v}_{i}}{d t}=\sum_{j=1}^{k_{i}}\left(\mathbf{f}_{c, i j}+\mathbf{f}_{d, i j}\right)+m_{i} \mathbf{g}$

Where $m_{i}$ and $\mathbf{v}_{i}$ are the mass and velocity of particle $i$ respectively. $k_{i}$ is the number of particles in contact with particle $i . \mathbf{f}_{c, i j}$ and $\mathbf{f}_{d, i j}$ are the contact force and viscous damping contact force respectively between particles $i$ and $j$. The final term $\mathbf{g}$ in Equation 1 is the force due to gravity. This model assumes that no other non-contact forces are acting on the particle. It is possible to include non-contact forces such as cohesive liquid forces in DEM models (Xu et al., 1999). The rotational motion of the particle can be calculated from:

$I_{i} \frac{d \boldsymbol{\omega}_{i}}{d t}=\sum_{j=1}^{k_{i}}\left(\mathbf{T}_{i j}+\mathbf{M}_{i j}\right)$

Where $I_{i}$ is the moment of inertia of the spherical particle $i$ given by:

$I_{i}=\frac{2}{5} m_{i} r_{i}^{2}$

Where $\boldsymbol{\omega}_{i}$ is the angular velocity of particle $i . \mathbf{T}_{i j}$ is the torque generated by the contact between particles $i$ and $j$ and $\mathbf{M}_{i j}$ is the rolling friction. The particle $i$ has radius $r_{i}$. The DEM modelling technique has three stages: contact detection; evaluation of contact forces; summation of forces to calculate particle motion. Contact detection is concerned with identifying if a contact has occurred between two particles, or a particle and any equipment geometry which may exist in the system. Evaluation of contact forces calculates the forces resulting from a single contact using an appropriate contact model. The resultant force is calculated by resolving all forces acting on a particle, including gravitational effects. Once this is determined Newton's second law of motion can be used to calculate the particle acceleration.

Rolling friction is responsible for bringing rolling objects to a rest. It is important to incorporate rolling friction into DEM especially if the particles are spherical. Rolling friction results from the elastic hysteresis loss as the rotating particle contacts other objects or as a result of any time dependent surface deformation it may experience (Tabor, 1952). Rolling friction has been successfully included in DEM contact models, such as Zhou et al. (1999) in simulation of sand pile formation. This work utilises the methodology of Xu et al. (2001) to include rolling friction. In this model the magnitude of the rolling friction torque, $\mathbf{M}_{i j}$, in Equation 4 is calculated by shifting the location of the normal contact force $\mathbf{f}_{c n}$ a distance $\boldsymbol{\delta}$ away from the contact point.

$\mathbf{M}_{i j}=\mathbf{f}_{c n} \times \boldsymbol{\delta}$

For a spherical particle contacting (and overlapping) a horizontal surface $\boldsymbol{\delta}$ is the horizontal distance between the centre of the particle and the where one side of the particle contacts the surface.

The selection of the numerical scheme used to calculate the particles' translational and rotational motion is important in the DEM. A balance must be found between computationally efficient and numerically accurate schemes. The original work of Cundall and Strack (1979) used an explicit time integration scheme. This explicit scheme is simple to implement and computationally efficient. 
However, the main drawback is that contacting particles' overlaps are not calculated until a time step has been completed. This requires small time steps to be used to ensure that particle overlaps are not over estimated and fictitious elastic energy during contacts are stored (Xu and $\mathrm{Yu}, 1997)$.

\section{Model Development}

The DEM model used for this research was developed from an existing 2D code used to study particle behaviour in fluidised beds (Xu and $\mathrm{Yu}, 1997)$. The code was updated to a 3D version and further developed during this work to include geometry representation via a finite element mesh and moving boundaries. The contact model used for this code is a linear spring dashpot model in the normal direction. An additional slider is incorporated in the tangential direction. There are many other nonlinear contact models available such as the Hertz (1882) and elastic-plastic models (Walton and Braun, 1986 and Thornton, 1997). Although these complex models were developed to more accurately represent contact physics within the system, they are still far from perfect requiring numerous simplifications with increased computational time. It has also been shown that more complex models are often no more accurate than linear models (Di Renzo and Di Maio, 2004).

\subsection{Representation of 3D geometry}

The geometry of the $250 \mathrm{ml}$ Mi-Pro was designed and built in SOLIDWORKS. Once the CAD model was complete a finite element mesh of the model was required. A mesh of the entire CAD model was not necessary for the DEM code; only the active surfaces inside the granulator would be required. These surfaces consist of the entire internal surface of the granulator's bowl and all the surfaces of the impeller. A finite element surface mesh is a collection of linked small elements which together can be used to represent a surface area. The software Gambit was chosen to create the finite element mesh of the Mi-Pro's geometry. Triangular elements were selected for the mesh as they only have three vertices (nodes); this is the least amount of nodes an element can have. The minimum number of nodes is desirable as less information is required to place an element into the 3D DEM code, reducing the computational load. The number of elements chosen was 428 for the bowl and 868 for the impeller. These numbers were selected so that they accurately represent the geometry, without having too many elements, which would increase the computational load. Simulations were performed with a larger number of elements and it was found that increasing the number did not affect the results for particle flow or contacts. Once the mesh was completed a mesh test was performed. This identified elements whose shape were furthest from an equilateral triangle and might affect the quality of the mesh.

\subsection{Contact detection}

Contact detection is one of the most important stages in any DEM model and often the most computationally expensive part of the procedure. In a system of $N$ particles, the search problem is of size $O\left(N^{2}\right)$ as theoretically each particle could interact with every other particle in the system. To reduce this number different contact detection techniques have been developed. Contact detection can be split into two steps: spatial sorting and contact resolution. Spatial sorting is concerned with reducing the complexity of the search problem by eliminating contacts deemed impossible in a single time step. Contact resolution defines when a contact has taken place. There are numerous different techniques available for the spatial sorting stage of the contact detection.

The two most common approaches for spatial sorting are the near neighbour method and the boxing or zoning method. Most codes employ either one of these techniques or a combination of both. The near neighbour technique works by defining a critical value $a$. This is set much larger than a value $b$ which is the distance beyond which contact cannot be achieved. The value of $b$ could be set as twice the largest radii of the particles in the system, or the maximum distance a particle can travel in a time step, if the maximum velocities of the particles are known. For each particle a list of its neighbours is created. This list comprises of all particles whose mass centre is located less than the distance $a$. The neighbour lists are updated once the given particle has travelled the distance $b$. Most models will have a relationship between the values $a$ and $b$ of the form: $a=k_{a b} b$, where $k_{a b}$ is a user defined constant. Typical values of $k_{a b}$ are around 1.5. The larger the value of $k_{a b}$ the less often the near neighbour list 
needs to be updated, but the longer the actual lists will be. Hoomans et al. (2000) used a near neighbour list in their modelling of gas fluidised beds as did Langston and Tüzün (1994) in their work on particle flows in hoppers. The zoning technique works by dividing the system domain into equal sized cubic boxes. The size of these cubes can be set as the value of the largest radii of the particles in the system or the value $a$ if it is to be used in conjunction with the near neighbour technique. Each particle is assigned to a cube (often referred to as cell) in which its centre lies. For each particle the model only searches for contact with other particles within that cube and the 26 cubes surrounding it in three dimensional space. Once the boxing process has been performed the near neighbour technique could be employed, but only particles within the boxing region of the particle under consideration will be searched for. Asmar et al. (2002) used both methods in their modelling work on particulate systems, and Mishra (2003) used the boxing method in their modelling work on tumbling mills. Iwai et al. (1999) proposed more efficient boxing methods known as, level-boxing, multi-level boxing and indexed-level boxing in their work on particulate simulations. Each of these techniques has their own advantages depending on how dense or sparse the particle assembly is and the range of particle sizes in the systems.

The contact resolution stage is a simple process for contact between spheres. This can be calculated if the location of the two spheres centres, their radii and the distance between them is known. For contact between a sphere and triangular element the contact detection becomes more difficult. For this work the finite wall method of Kremmer and Favier (2001a) was used to determine if contact occurred between the spherical particles and the triangular element. For the finite element method the triangle is defined by three corner points, $\mathbf{n o}^{1-3}$ in the global coordinate system (Figure 1).

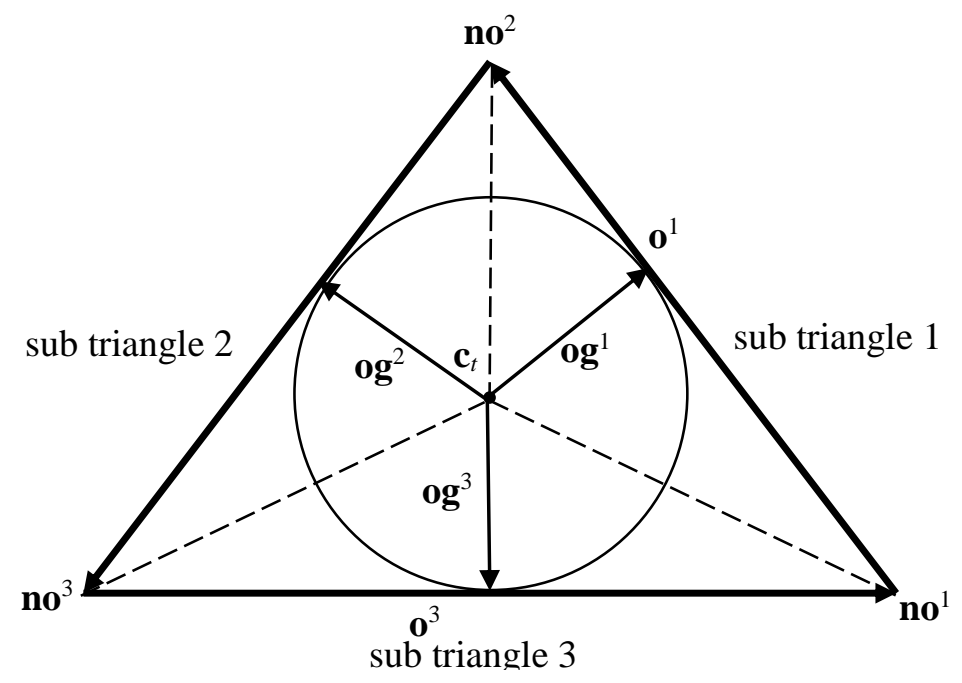

Figure 1: Wall element description vectors, adapted from Kremmer and Favier (2001a).

Vectors which represent the sides of the triangles are calculated from the corner points; these are labelled $\mathbf{o}^{1-3}$ and are in a coordinate system local to the individual wall element. Three vectors $\mathbf{o g}^{1-3}$ are defined; these are orthogonal to the corresponding wall element sides, all equal in length and in the local coordinate system. A surface element reference point $\mathbf{c}_{t}$ is found from vector mathematics. This is a point at the centre of a circle which inscribes the wall element and is in the global coordinate system. The wall element is given a virtual radius of curvature $\left(r_{c}\right)$, this is used in the contact detection and is taken from the curvature of the initial geometry. A unit vector $\left(\mathbf{u c}_{t}\right)$ which is normal to the elements surface and starts at the surface element reference point is defined. The particle is spherical in shape and defined by a centre point $\left(\mathbf{c}_{s}\right)$ and a radius $\left(r_{s}\right)$. Planar penetration is detected by first creating a vector $\left(\mathbf{v}_{\mathrm{c}}\right)$ from the wall element reference point $\mathbf{c}_{\mathrm{t}}$ to the sphere centre point $\mathbf{c}_{\mathrm{s}}$ :

$\mathbf{v}_{\mathrm{c}}=\mathbf{c}_{\mathrm{s}}-\mathbf{c}_{\mathrm{t}}$ 
The vector $\mathbf{v}_{\mathrm{c}}$ is then projected onto the wall element normal vector $\mathbf{u c}$. The result is a vector $\mathbf{p p}$ which is normal to the wall element with a magnitude equal to the distance between the wall element and the sphere's centre. The sphere's radius $\left(\mathbf{r}_{\mathrm{s}}\right)$ is subtracted from $\mathbf{p p}$, if the result is less than zero planar penetration has occurred and the next step of contact detection is performed. By utilising the coordinates and vectors defined in Figure 1 it is possible to calculate whether contact detection has occurred at the surface of the element or one of its edge or vertices. The contact detection algorithm for the finite wall method can be view in the flow diagram in Figure 2.

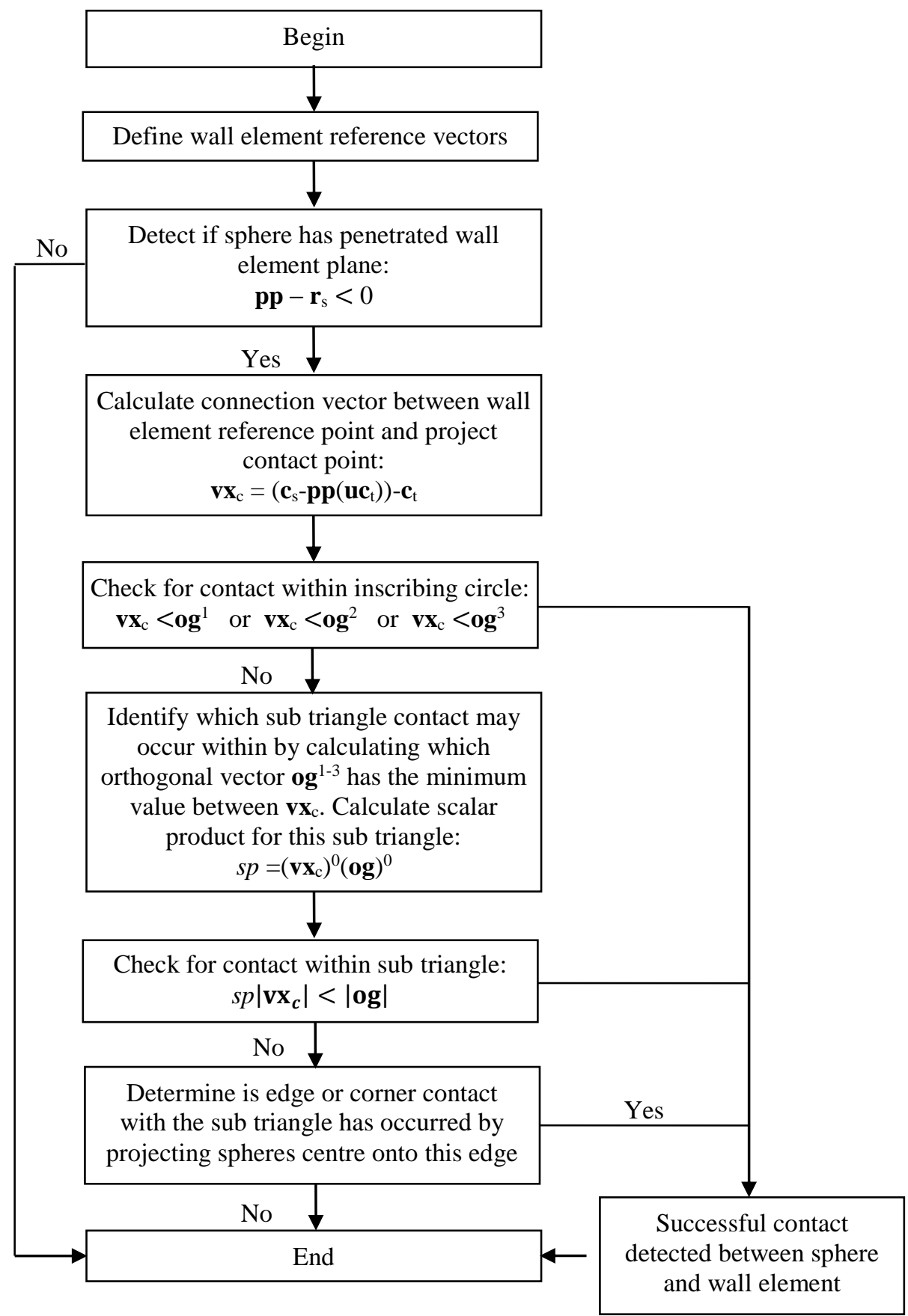

Figure 2: Flow diagram for contact detection using the finite wall method.

Wall elements are connected together along edges, and this presents a problem. If a sphere is in contact with the edge of one element it must also be contacting another element. To overcome this problem in the finite wall method the wall elements are shrunk by a fraction of the sphere's radius so that they no longer act as connected elements and contact with the correct element can be identified. The value that the elements are shrunk by must be carefully selected so that contact is still detected 
with one of the elements. Kremmer and Favier (2001a) recommend shrinking the wall elements less than $0.5 \%$ of the sphere's radius, and a value of $0.3 \%$ was used in this work. The DEM code included algorithms to detect if contact is detected with two connected parallel elements. For all the simulations reported in this thesis no contact was detected for two parallel connect elements and it is concluded that the element shrinkage method was successful.

\subsection{Moving Geometries}

The Mi-Pro granulator has a moving impeller. To include the motion of this in the DEM model the surface mesh of the impeller must be able to move. To implement motion of the surface mesh of the impeller, each element in the mesh was moved at the end of each simulation time step. This was performed by moving the location of each of the element's nodes. The motion of the impeller is rotational around the $\mathrm{y}$ axis; therefore only the $\mathrm{x}$ and $\mathrm{z}$ coordinates of the nodes were changed.

As the motion is rotational, the node positions were switched from a Cartesian coordinate system to a Polar coordinate system. This is a system where every node is defined as a distance $r$ from a centre point and an angle $\theta$ from a fixed line which runs through the centre point Transposing a point from a Cartesian coordinate system to a Polar system is a mathematical task performed using trigonometry.

Once the nodes were located in a Polar coordinate system they could be moved to their new location by $\theta_{I}$, this is the angle that the impeller moves through in a time step and was calculated using the equation below:

$\theta_{I}=\frac{2 \pi(R P M) \Delta t}{60}$

Where $R P M$ is the impeller's rotational speed in revolutions per minute and $\Delta t$ is the time step of the simulation. Once a new angle from the reference line was calculated for all of the nodes their new position in the Cartesian coordinate system was calculated. The wall element description vectors were then redefined with the same convention as Figure 4. When a DEM code has moving geometry it is not just the location of the elements that needs to change in the code. The equations used to resolve contacts must be altered to account for a contact between a particle and a moving element. This is straight forward procedure which simply requires the element to be given a velocity. The velocity of the element $v_{e}$ must be resolved into components in the $x$ and $z$ directions, as these are the directions in which the element experiences motion. The magnitude of the velocity $v_{e}$ is found by using the equation for velocity of a point in circular motion:

$v_{e}=\frac{2 \pi(R P M) r_{d}}{60}$

Where $r_{d}$ is the distance of the point from the axis of rotation. For the elements the point used to calculate the velocity is the wall element reference point ( $\mathbf{c}_{t}$ in Figure 4$)$. Once the velocity of the element is calculated, trigonometry can be used to calculate its components in the $x$ and $z$ directions. For computational efficiency the wall element is assumed to have the same velocity across its entire surface.

\subsection{Selection of input parameters}

The correct selection of simulation parameters for any numerical modelling technique is important and DEM is no exception. Inputs that represent process and material variables such as impeller speed, particle size, and density are simple to include. Other variables require careful consideration and calculation before their value can be determined. For the DEM these variables are contact model spring stiffness, contact model damping coefficient, simulation time step and contact model sliding friction coefficient. The methodology chosen for selecting these parameters is discussed below. 
Arbitrary values were selected for the particle properties so that the results could be compared to the literature. The particle radius and number was selected to accurately represent granules flowing in a granulator. $1 \mathrm{~mm}$ was chosen as a suitable particle radius size and a particle number of 18,000 was selected to result in a volume of granules representative of a lab scale granulation. Particle number is a critical parameter of DEM models as the number of particle directly affects the simulation time due to the number of contacts which must be resolved each time step. The particle density was selected as a representative value for the density of solid granules. The value of 0.3 was chosen for the particle particle and particle - geometry frictional coefficient. This value is consistent with those used in the literature and resulted in particle tangential velocities comparable with ones recorded from the validation experiments. The impeller speed of 250 RPM was chosen as this is the speed the granulator often operates at and a common value used in the literature.

\subsubsection{Spring constant}

The selection for the value of spring constant $k$ is critical as it directly affects the amount of inter particle overlap and simulation time step $\Delta t$. If the value of spring constant selected is too small it can lead to large overlaps, which could affect bulk parameters such as coordination number (Di Renzo and Di Maio 2004). Researchers still use small $k$ values in their DEM models as it allows for larger simulation time steps; as $\Delta t$ is usually calculated using the formula for a single degree of freedom system original proposed by Cundall and Strack (1979). There are many reported uses of linear models in the literature, with many different values of $k$ used. For work on fluidised beds many authors use a low value of $k$ often around 800N/m (Tsuji et al., 1993; Mikami et al., 1998; Rhodes et al., 2001). They justify the use of such a small value by the fact that fluid drag forces are the predominant forces in these systems and not contact forces. High shear granulators are a different type of equipment where motion is caused by moving impellers. In these systems particle contacts are critical to the motion and state of the system. Therefore, the use of a low value for spring stiffness is not suitable. Cleary (2000) suggested that particle overlaps should be in the range of $0.1 \%-1 \%$ of the particle's diameter. This would correspond to spring constants of around $10^{6}-10^{7} \mathrm{~N} / \mathrm{m}$. In later simulation work on tumbling mills Cleary and Hoyer (2000) investigated the effect of a varying spring constant and found that the value of $10^{7} \mathrm{~N} / \mathrm{m}$ gave a mean overlap of $0.6 \%$ and a maximum overlap of $15 \%$ of the particles diameter. The flow patterns produced from the simulations were also similar to ones recorded experimentally using high speed photography. In the literature many DEM models of mixers and granulators using linear contact models have been presented. The DEM models of Terashita et al., 2002; Sinnott and Cleary, 2003; Kuo et al., 2004; Sato et al., 2008, have a variety of different geometries operating at a range of impeller speeds. This would result in many different contact velocities, yet none reported the maximum or average overlaps recorded from their results or justified their choice of spring constant. Kuo et al. (2004) used a range of spring constants in their simulation. Their model geometry was a cylinder fitted with either a rotating disc or a flat horizontal blade. When the disc was fitted their results showed that the larger spring constant gave overall better agreement with particle velocities obtained from PEPT experiments. However, the particle velocities calculated from the simulations still varied greatly from the PEPT results. The results for the simulations when the paddle was fitted gave very unrealistic particle flow, so it would be hard to draw any meaningful results from the effect of varying spring constant.

It appears that the selection for the spring constant value depends very much upon the application of the model. Mishra and Murty (2001) derived an equation for calculating the value for spring constant if the maximum acceptable overlap and maximum contact velocity in a system is known:

$$
k=\frac{f^{2} m v_{0}^{2}}{d^{2}}
$$

Where $m$ is the mass of a typical particle in the system, $v_{0}$ is the maximum velocity of a typical particle in the system. $f$ is the penetration factor, which is a typical particle's diameter $d$ divided by the desired overlap. As the maximum particle velocity in the Mi-Pro granulator model could be taken as 
the maximum tip speed of the impeller, the above equation can be used to calculate suggested values for spring constant for the impeller speed and inter particle overlap values. Figure 3 shows the $k$ values calculated from the above Equation 8, for particles of radius $1 \mathrm{~mm}$ and density $641 \mathrm{~kg} / \mathrm{m}^{3}$. Calculations were made for the maximum velocities from the corresponding impeller speeds. The maximum velocities were calculated using Equation 7 for impeller tip speed where $r_{d}$ was selected as the impeller radius.

Figure 3 indicates that if particle overlaps of less than $1 \%$ are desired, the spring constant $k$ should be at least 10000. Therefore, for the simulations of particle flow in the Mi-Pro granulator a value of $10000 \mathrm{~N} / \mathrm{m}$ was selected for the spring constant. From the theoretical results presented above this should result in overlaps which are inside the recommended range suggested by Cleary and Sawley (2002), and should not produce results with unrealistic particle contact mechanics or motion.

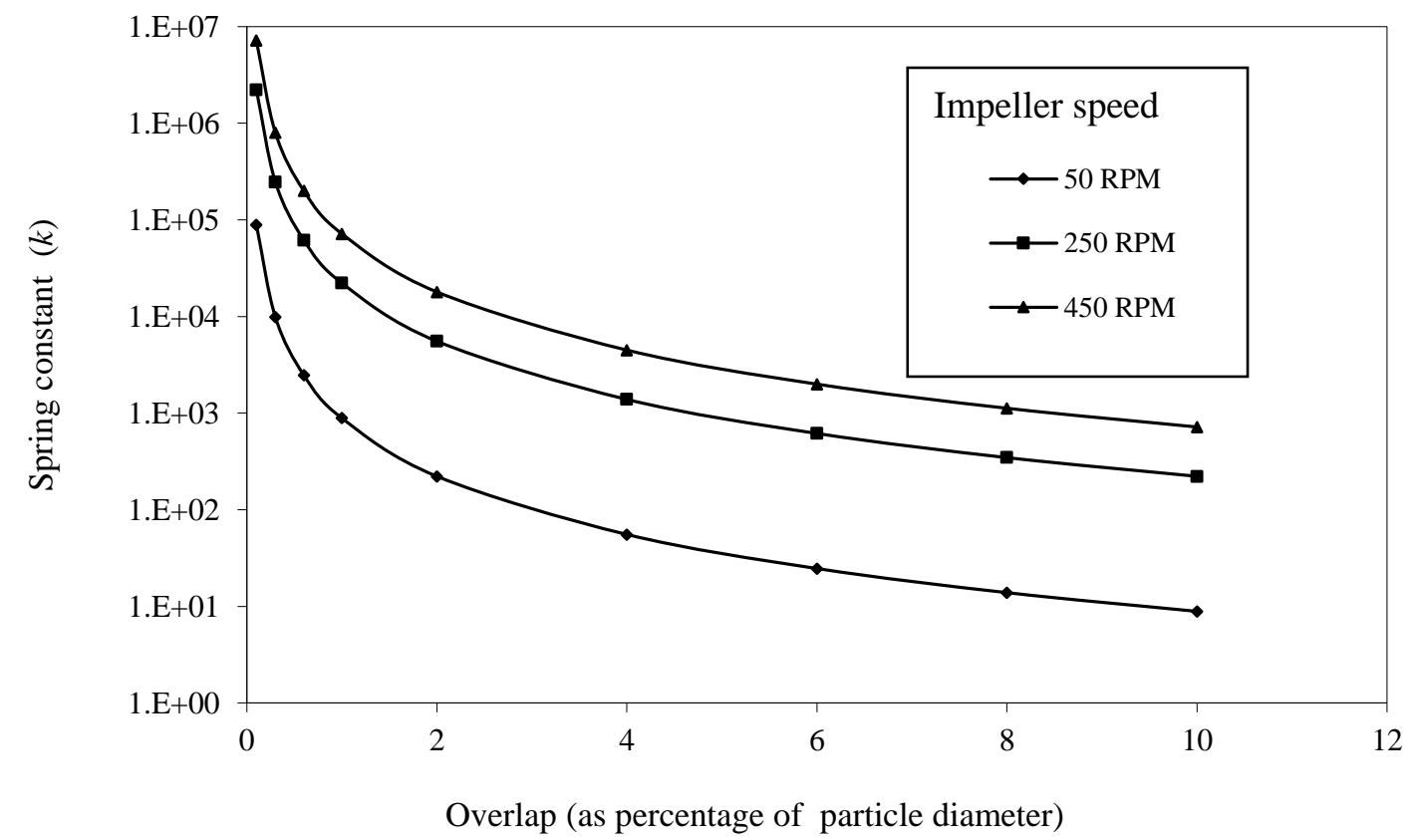

Figure 3: Calculated spring constant values for a range of maximum allowable overlaps and particle velocities (calculated based on maximum impeller tip speed).

\subsubsection{Damping coefficient}

The damping coefficient is determined by the contact properties of the particles and can be related to the coefficient of restitution $(e)$.

$\eta=\frac{2(\ln e) \sqrt{m k}}{\sqrt{(\ln e)^{2}+\pi^{2}}}$

A dashpot is used to represent viscous damping during contacts which would result from plastic deformation and other energy loses. The viscous damping is represented as a force which is calculated by a damping coefficient multiplied by the relative velocities of the contacting particles. The coefficient of restitution for contacting particles can have a value between zero and one. Zero represents a contact where all the kinetic energy is dissipated and the particles stop bouncing back, whereas one represents a totally elastic contact where all of the kinetic energy is preserved. Wang and Mason (1992) define the coefficient of restitution as: 
$e=\left|\frac{v_{a}}{v_{b}}\right|$

Where $v_{a}$ and $v_{b}$ are the normal components of the particle velocity before and after the contact. The coefficient of restitution is not just dependent upon the contacting particle properties but also the relative speed of the contact (Kuwabara and Kono, 1987). Despite this, many DEM modellers take $e$ as a constant for all impact velocities. Labous et al. (1997) performed experimental work and showed that the value of $e$ was only sensitive to change for contact velocities over $10 \mathrm{~m} / \mathrm{s}$. This value is significantly greater than any velocities the particle will be experiencing in the Mi-Pro granulator so for the simulations performed during this research it is assumed that $e$ is constant.

$\mathrm{Xu}$ and $\mathrm{Yu}$ (1997) performed simple simulations of a single particle dropped from an initial height $h_{0}$ against a flat surface. This surface had identical properties to the particle. For the single drop simulations the effect of gravity was not considered whilst the particle was in contact with the flat surface. This was to ensure that the only loss of energy would be as a result of the damping term in the contact model. They recorded the height that the particle would rebound to $h_{l}$ and used the equation below to calculate the coefficient of restitution for different damping coefficients.

$$
e=\sqrt{\frac{h_{1}}{h_{0}}}
$$

Theoretically if the coefficient of restitution for a contact is known the damping coefficient $\eta$ can be calculated from (Ting et al 1989):

$\eta=\frac{2(\ln e) \sqrt{m k}}{\sqrt{(\ln e)^{2}+\pi^{2}}}$

Where $m$ and $k$ are the mass and spring constant used in the contact model. The equation can be derived by solving the equation of motion for a mass-spring-dashpot system (Malone and $\mathrm{Xu}, 2008$ ).

To select the most appropriate value for the damping coefficient, simulations were performed similar to the work of $\mathrm{Xu}$ and $\mathrm{Yu}$ (1997). A single particle dropping from a height against a flat surface was simulated. The height it returned to was recorded. This was repeated for various damping coefficients. The height the particle returned to was used to calculate $e$. The other simulation parameters can be seen in Table 1. The results from these simulations were compared with theoretical results from Eq 12.

Table 1: Simulation parameters for coefficient of restitution calculations.

\begin{tabular}{|l|l|}
\hline Particle radius $r_{p}$ & $0.001 \mathrm{~m}$ \\
\hline Particle density $\rho_{p}$ & $641 \mathrm{~kg} / \mathrm{m}^{3}$ \\
\hline Spring constant $k$ & $10000 \mathrm{~N} / \mathrm{m}$ \\
\hline Simulation time step $\Delta t$ & $3.42 \times 10^{-6} \mathrm{~s}$ \\
\hline Drop height $h_{l}$ & $0.03 \mathrm{~m}$ \\
\hline
\end{tabular}


From Figure 4 it can be seen that the simulated and theoretical results show good agreement. It was concluded that equation 11 is satisfactory for calculating the damping coefficients required and was used for the modelling work.

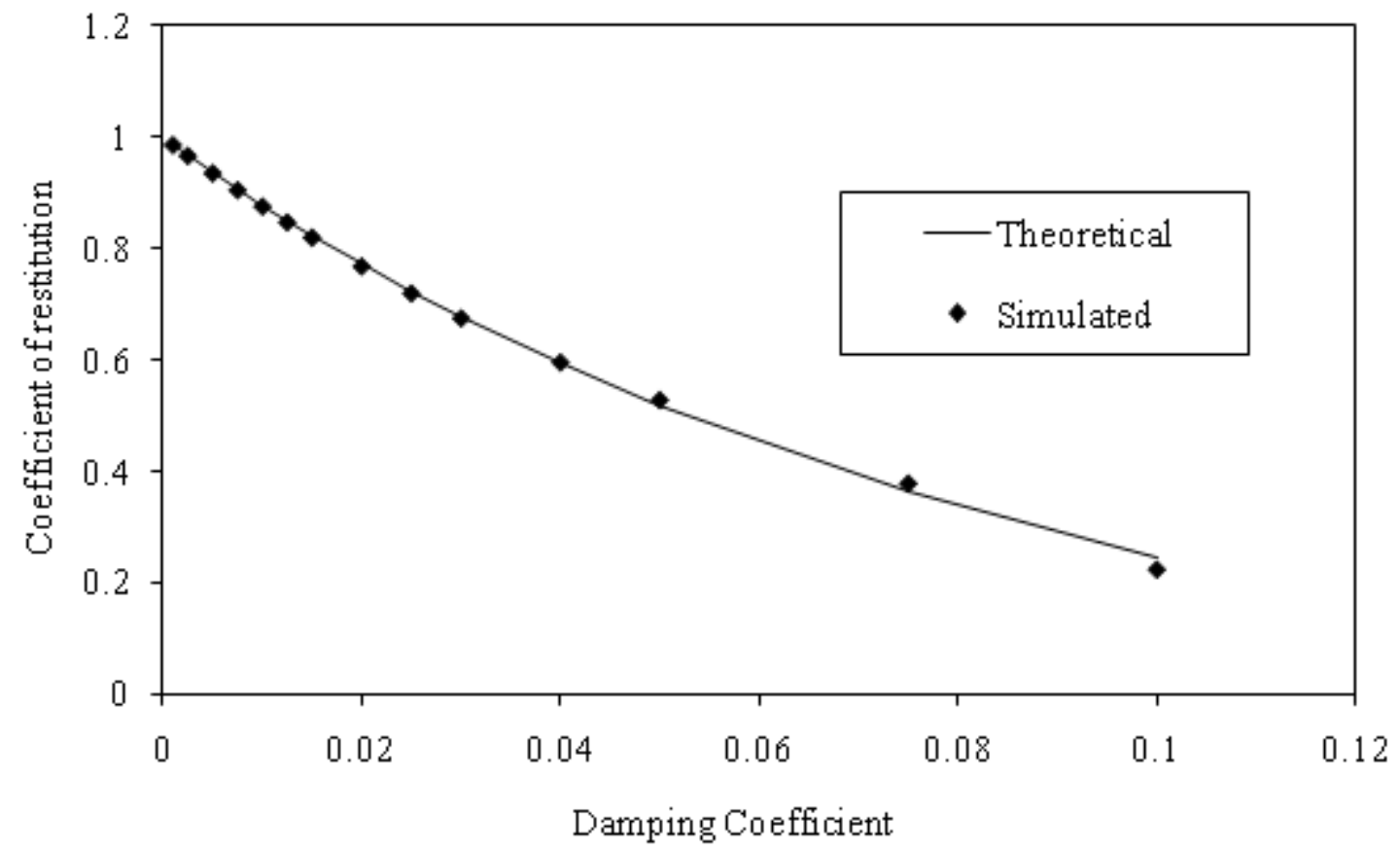

Figure 4: Theoretical and simulated results for the coefficient of restitution for different damping coefficients.

\subsubsection{Time Step}

The simulation time step directly affects the computational time to perform a DEM simulation. As already mentioned DEM simulations are computationally expensive, so anything to reduce this load is advantageous. One of the underlying assumptions of DEM is that in a single time step the disturbance of a particle contact cannot propagate any further than its nearest neighbours, for this to hold true the time step must be small. If the time step is too small simulations will take too long to complete, whereas if the value is too large contact dynamics will be jeopardised and the integrity of the model will come into question. The original DEM work of Cundall and Strack (1979), they suggested that the simulation time step should be some fraction of a critical time step:

$$
\Delta t_{c r i t}=2 \sqrt{\frac{m}{k}}
$$

The above equation is based on the natural frequency of a single degree of freedom oscillating system. Which in this case is a mass $m$ connected to a spring $k$. This work does not suggest what would be a suitable fraction of the critical time step though. Most DEM simulations use a proportion of the critical time step presented in the form:

$$
\Delta t=C \sqrt{\frac{m}{k}}
$$

Where $C$ is a constant, some examples of values chosen for $C$ include: 0.4 (Ramos et al., 1998), 2 (Rajamani et al., 2000), 0.2 (Mishra and Murty, 2001) and $\pi / 5$ (Kuo et al., 2002). 
$\mathrm{Xu}$ and $\mathrm{Yu}$ (1997) performed particle dropping simulations to determine the value for their time step. They decided to consider energy conservation. In their simulations they dropped a particle from a known height, allowed it to contact a flat wall without viscous damping, and recorded the height it returned to. They concluded that the ideal time step would be the largest one with which the particle would return to exactly the same height. For the selection of the value of time step used in this work the methodology of Xu and $\mathrm{Yu}$ (1997) was adopted. Simulations were performed with the dropping of particles against a flat surface. The simulation parameters can be seen in Table 1 and the drop height $h_{1}$ was $0.042 \mathrm{~m}$. The height the particle was dropped from $\left(h_{0}\right)$ and the height it returned to after the first contact with the flat bottom wall $\left(h_{r}\right)$ were used to calculate the percentage error in energy:

$$
\left(1-\left|\frac{h_{r}}{h_{o}}\right|\right) \times 100
$$

This percentage error in energy was calculated for each value of $C$. Whilst the particles were in contact with the wall viscous damping was not considered in the contact model. This would ensure that the error in the energy was only affected by the simulation time step. The results from the simulations can be seen in Figure 5.

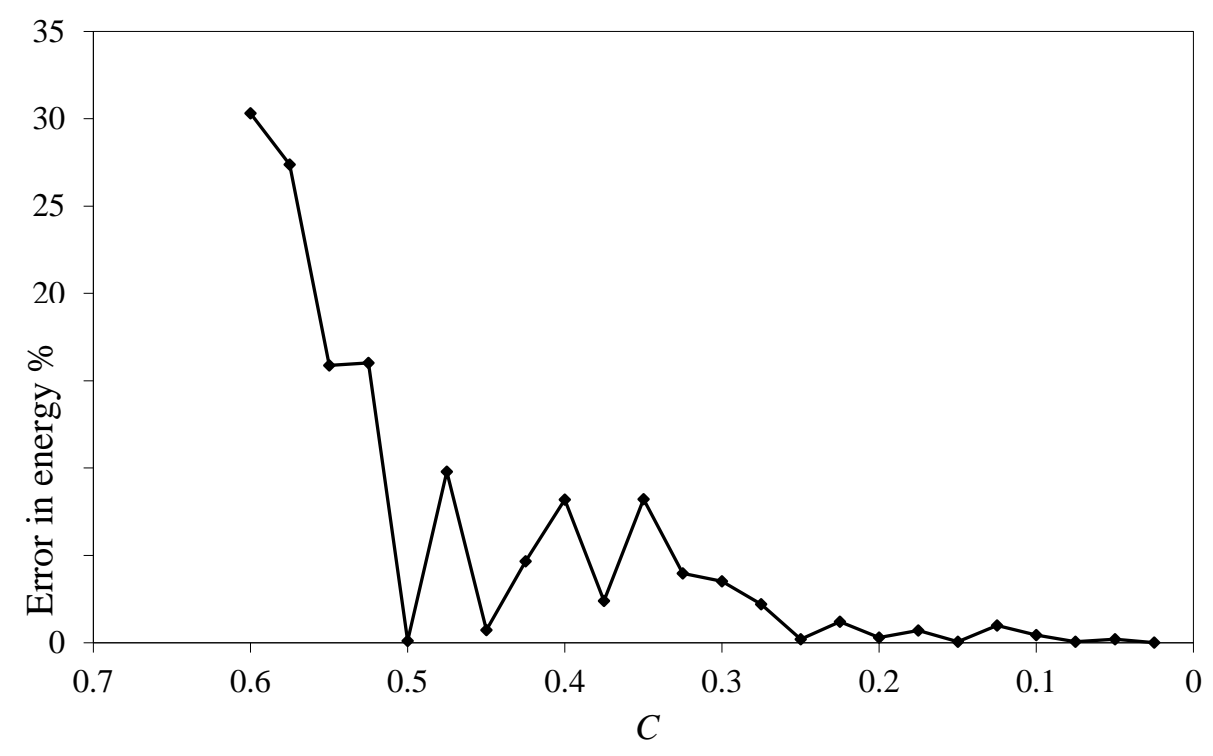

Figure 5: Percentage error in energy against $C$ (proportion of critical time step).

Figure 5 shows that as $C$ decreases the percentage error in energy decreases. However, there are some fluctuations in the results. At a value of $C=0.1$ the error in energy is small and decreasing $C$ further shows no great improvement. Therefore, 0.1 was the value chosen for $C$ in the simulations presented in this work.

All of the simulation parameters for the following results presented in section 4 of this work can be seen in Table 2:

Table 2: simulation input parameters. 


\begin{tabular}{|l|l|}
\hline Parameter & Value \\
\hline Particle radius $r_{p}$ & $1 \mathrm{~mm}$ \\
\hline Number of particles & 18,000 \\
\hline Particle-particle friction coefficient $\gamma$ & 0.3 \\
\hline Particle-geometry friction coefficient $\gamma$ & 0.3 \\
\hline Particle density $\rho_{p}$ & $1750 \mathrm{~kg} / \mathrm{m}^{3}$ \\
\hline Coefficient of restitution $e$ & 0.9 \\
\hline Simulation time step & $5.41 \mathrm{e}^{-6} \mathrm{~s}$ \\
\hline Spring constant $k$ & $10,000 \mathrm{~N} / \mathrm{m}$ \\
\hline Impeller speed & $250 \mathrm{RPM}$ \\
\hline
\end{tabular}

\subsection{Simulation Procedure}

To begin the simulation the 18,000 particles were arranged into a block above the impeller blades. A rectangular hole was removed from this block to allow for the location of the impeller shaft. The particles were then allowed to fall under gravitational forces and settle in the bowl. The impeller was then rotated 360 degrees forward and then 360 degrees backwards at a speed 60 RPM. This was the starting position of the particles for all simulations. The impeller motion was then started again at its selected speed and all simulations were performed for a duration of $10 \mathrm{~s}$. One of the primary benefits of using DEM is that dynamic information at the particle scale can be found. This does present an issue though with the amount of data that will be generated from a simulation. For the simulations reported in this article data was saved every $1 \mathrm{~ms}$. The following data was saved for each particle: location in Cartesian space, velocity and forces acting on particle. This data was found to be sufficient to be able to investigate the particle flow in the Mi-Pro.

\subsection{Model validation}

To validate the DEM model of the Mi-Pro dry mixing experiments were performed and particle motion was recorded using a high-speed video camera (Vision Research Phantom V710). This was gratefully loaned from EPSRC equipment loan pool. The Mi-Pro has transparent bowl walls so it was possible to view and record the particle motion there. APG Molsiv adsorbent beads were used to represent dry particle motion. These beads were coated with magnesium stearate to improve their flowability and prevent trapping under the blades. The $250 \mathrm{ml}$ bowl was filled with $50 \mathrm{~g}$ of these beads and 10 seconds of mixing at 250 RPM was recorded. Colored tracer particles were placed in the system and average tangential velocities, for particles at the side of the bowl, were calculated. The APG beads have an average diameter of $2 \mathrm{~mm}$ and a bulk density of $641 \mathrm{Kg} / \mathrm{m}^{3}$. These were selected as they had a comparable size to the DEM experiments and would not experience any breakage or electrostatic effects. In order to choose appopriate values for simulation parameters, especially related to frictional interactions, simulations were performed with various values of coefficient of friction for particle-particle and particle-wall interactions. Figure 6 shows an image recorded from the particle flow experiments, the black coloured beads can clearly be distinguished from the uncoloured ones. 


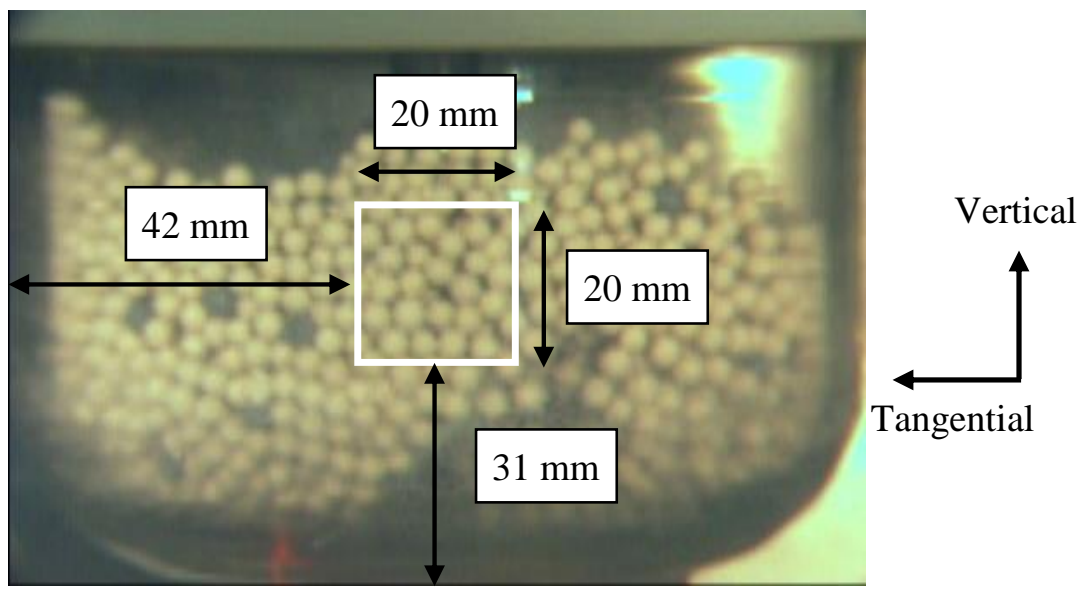

Figure 6: Image of bead flow at 250 RPM in the Mi-Pro granulator. The white squares is the velocity calculation cell.

From the view of the side of the bowl, the beads' flow in the vertical and tangential directions were observed and calculated. The tangential direction is defined as motion in the same direction as the rotating impeller, in the two dimensional image this appears as the horizontal direction. A white square cell was superimposed onto the images and the mean tangential velocity for this cell was calculated. The sides of the square cell were $20 \mathrm{~mm}$; this value was selected as it allowed a reasonable average to be calculated in an efficient manner. The mean tangential velocity of the beads across the cell was calculated for comparison with results from the DEM simulations. Tangential velocity was chosen as it was observed that the motion was predominantley in this direction. The beads did experience motion in the vertical direction as a result of the inclined blade forcing them upwards and gravity pulling them down. The direction and magnitude of this vertical motion was very much dependent on the beads location relative to the impeller's blades. The average tangential velocity of every coloured bead that passed across the cell was calculated. A mean tangential velocity for the cell was calculated from all of the coloured beads average velocities. The beads' average velocity $\left(v_{b}\right)$ across a cell was found using:

$v_{b}=\frac{C L}{\Delta t}$

Where $C L$ is the horizontal distance across the cell. This was calculated by measuring the length of the cell and the length of the image. The actual dimensions of the Mi-Pro's bowl were already known so the distance the cell represents could be calculated. When this distance was calculated care was taken to ensure it was the actual distance travelled by the particle. As the bowl is curved this is an arc and not a straight line as the $2 \mathrm{D}$ image might suggest. $\Delta t$ is the time it takes the particle to cross the cell. This was calculated by counting the number of images it took the particle to cross the cell and multiplying it by the time interval between two images $(0.002 \mathrm{~s})$. The mean tangential velocity of all coloured particles passing through the cell was then calculated.

Figure 7 indicates that a frictional coefficient of 0.3 for particle - particle and particle - geometry interactions results in comparable tangential velocities for the experiemental and simualtion results. The average tangential velocity from the simualtions was $0.446 \mathrm{~m} / \mathrm{s}$ and from the experiments 0.432 $\mathrm{m} / \mathrm{s}$, again showing good agreement. The friction between a particle and the wall has more pronounced effects than the friction among particles. The results from these experiments validate the model in respect to particle velocities in near wall regions. 


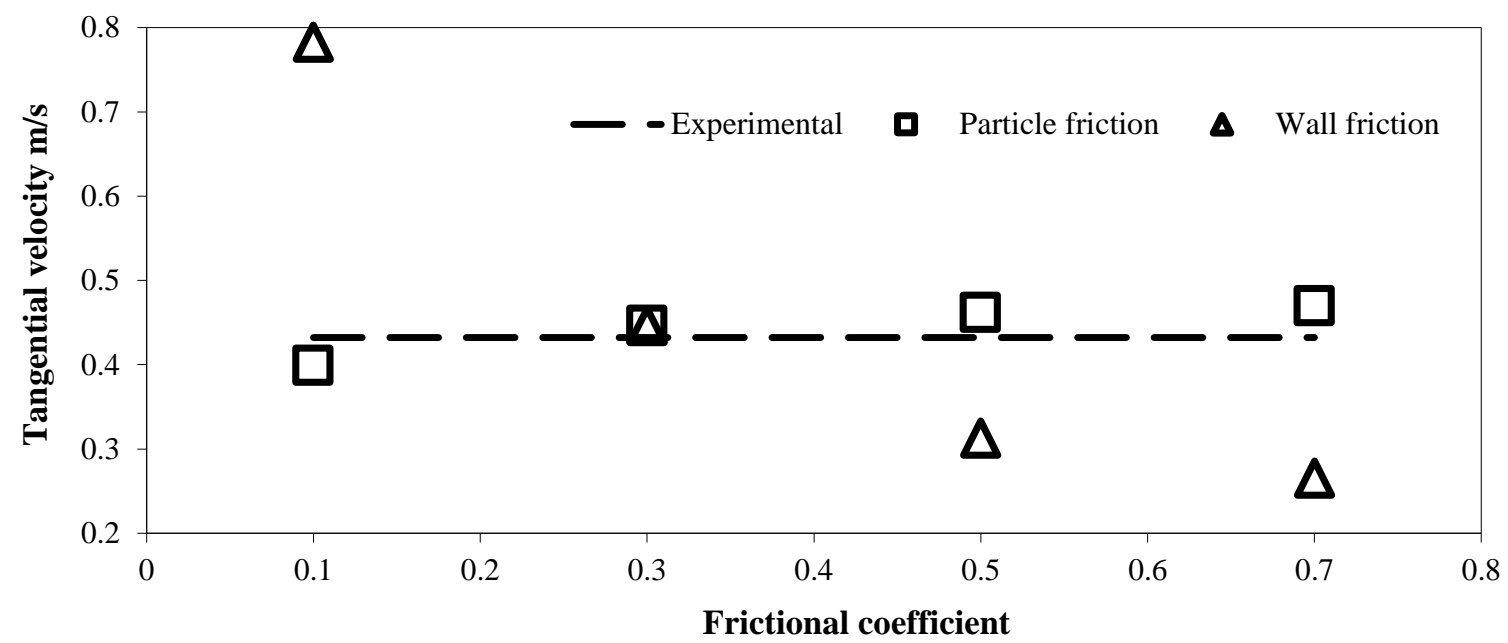

Figure 7: Validation results for near wall particle velocities in the Mi-Pro with an impeller speed of 250 RPM.

\subsection{Results processing}

This paper will present the simulation results from the Mi-Pro granulator as particle velocities and particle packing density. Particle velocities will be presented in two ways. Firstly velocity vectors averaged over space and time will be calculated; this is similar to the methodology used by Zhou et al. (2003). To calculate the time and space averaged velocities the domain of the Mi-Pro DEM model was split into cubic cells. For each cell a velocity vector was calculated by averaging the velocities of every particle present in a cell at each simulation save point. The cell velocity vectors will then be presented in horizontal and vertical sections so that the spatial distribution of the particle velocities can be studied. The horizontal sections will show motion in the tangential and radial directions, and the vertical sections will show motion in the vertical and radial directions. To calculate the velocity vectors in horizontal sections the simulation bed is split up into 12 horizontal sections with each one representing a different vertical height. Starting from the bottom of the bowl, each horizontal section has a height of $4.5 \mathrm{~mm}$. Each horizontal section is then split into a series of cells all with sides 4.5 $\mathrm{mm}$. The data used to calculate the average velocities is taken after five seconds of the simulation; this is when a steady state of motion is established. The average velocity for a cell is calculated by averaging the velocities of all the particles whose centres are located within the cell's boundaries at a save point. For each save point except the first, the particle's position and velocity had to be transposed in a rotational direction. This was to account for the motion of the impeller between save points and to ensure that their location was always relative to the impeller's position at the first save point.

The size of the calculation cells must be given careful consideration. If the cell size is too large the velocity vector will not give a fair representation of the particle flow within it. However, if it is too small the figures will have many vectors which may mask the general features of the flow. It is also possible that small cells may only have a few particles occupying them throughout the simulation and not enough to calculate a reasonable average velocity for that cell. Zhou et al. (2003) split their mixer domain into cubic cells with $12.5 \mathrm{~mm}$ sides. This created a grid of $20 \times 20$ cells for each horizontal section. However, they did not comment on why these cell sizes were selected. In this work the following cell sizes were tested: $3 \mathrm{~mm}, 4.5 \mathrm{~mm}$ and $6 \mathrm{~mm}$. The larger size $(6 \mathrm{~mm})$ did not display some of the features of the particle flow, especially around the impeller blades. The cell size of 4.5 $\mathrm{mm}$ shows all of the same features of the smaller cell size so were deemed suitable for this work. From initial observations of the simulated flow in the Mi-Pro granulator it was found that the majority of the motion was in the tangential direction. To give a greater insight into the motion the velocity vectors will be presented relative to the impeller's rotation. This is calculated by subtracting the rotational velocity of a point on the impeller, the same radial distance from the axis of rotation as the 
particle, from the velocity of the particle. The magnitude of the impeller's rotational velocity at a point, a radial distance from the axis of rotation $\left(v_{i r}\right)$ can be calculated using equation 7 . The direction of the velocity is in the same rotational direction as the impeller.

To calculate the velocity vectors in vertical sections the granulator was split into 36 wedge shaped sections. For each vertical section a grid of cells was created, the velocity for a cell was found by averaging the velocities of all particles in the cell. This was completed in the same manner as the velocity vectors for the horizontal sections. The selection of the size of the cells in the vertical sections followed the same procedure as described above for horizontal sections. The Mi-Pro has three identical impeller blades, giving it geometric symmetry. For this reason the velocity vectors in vertical sections were only calculated for one third of the bowl.

The tangential velocity of the particles at different radial distances gives information on how the particle velocities vary with radial distance from the bowl's centre. The tangential velocity at a radial distance is calculated by averaging the tangential velocity of all particles between certain radial distances. The tangential velocity of a single particle is the component of the velocity travelling in the same direction of the impeller and is calculated using the following equation:

$\mathbf{v}_{t}=\mathbf{v}_{z} \cos \alpha-\mathbf{v}_{x} \sin \alpha$

Where $\alpha=\arctan (z / x)$, and $x$ and $z$ are the position of the particle in a horizontal plane. $\mathbf{v}_{x}$ and $\mathbf{v}_{z}$ are the particle's velocity resolved into the $x$ and $z$ coordinates respectively. This tangential velocity is the absolute velocity of the particles and is not relative to the impeller blade's velocity.

Packing density displays where the particles are located in the system and how closely packed together they are. A value of packing density was calculated for each of the cells used to calculate an average velocity vector. The packing density for each cell was averaged with respect to time. The packing density is the inverse of the bulk porosity and was calculated using the following equation:

$P D=1-\frac{V_{c}-\left(n \times V_{p}\right)}{V_{c}}$

Where $P D$ is the packing density of a cell, $V_{c}$ is the cell volume, $n$ is the number of particles whose centre is located inside the cell and $V_{p}$ is the volume of an individual particle. It is possible that a particle whose centre is not located in the cell may have some of its volume located within it. It is also possible that a particle whose centre is located in a cell may not have its entire volume located in that cell. More complex equations for packing density were developed to account for these scenarios.

However, since the packing density for a cell is averaged over the entire simulation time, these were found to produce very similar results to the above equation so were not utilised. In some of the cells the geometry of the Mi-Pro's bowl was presented, this was taken into account when calculating the volume of that cell. Studying the packing density of the system will enable a deeper understanding of the flow structure as it will identify regions where particles are densely packed and regions where particles are less packed with more free space around them.

\section{Simulated particle flow in Mi-Pro}

The DEM model developed during this work was used to simulate particle motion in a Mi-Pro granulator. The simulations parameters can be seen in Table 2, the simulation procedure in section 3.5 and data analysis procedure in section 3.7.

Figure 8 displays four images of the particles in the Mi-Pro, at different times after the impeller motion has begun. Once the impeller starts moving it forces the particles immediately in front of each blade forwards and upwards (Figures 8a and b). This creates a wake region behind each impeller blade. The initial rotation of the impeller results in many particles located in the upper region of the bowl (Figure 8c). These particles then fall down under gravity and a steady state of motion is established (Figure 8d). Videos of the simulated particle motion indicated that the major component 
of the particle motion was in the tangential direction of the rotating impeller. The particles with the highest velocities were found to be immediately in front of the impeller's blades. Particle motion exists in three directions in the Mi-Pro granulator: tangential, radial and vertical.

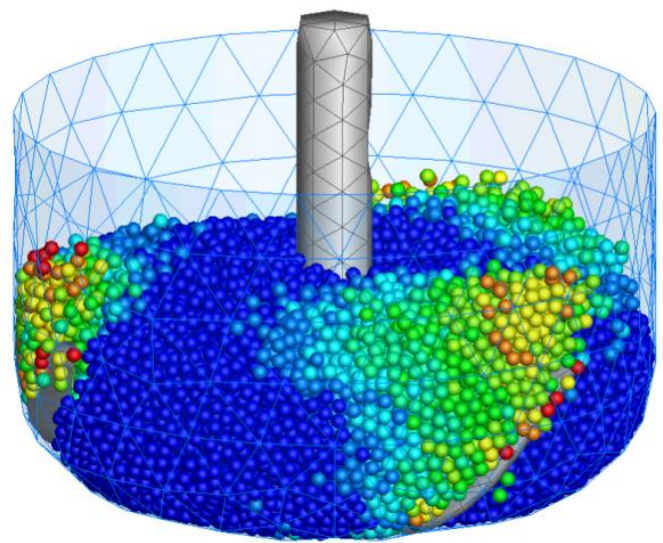

(a)

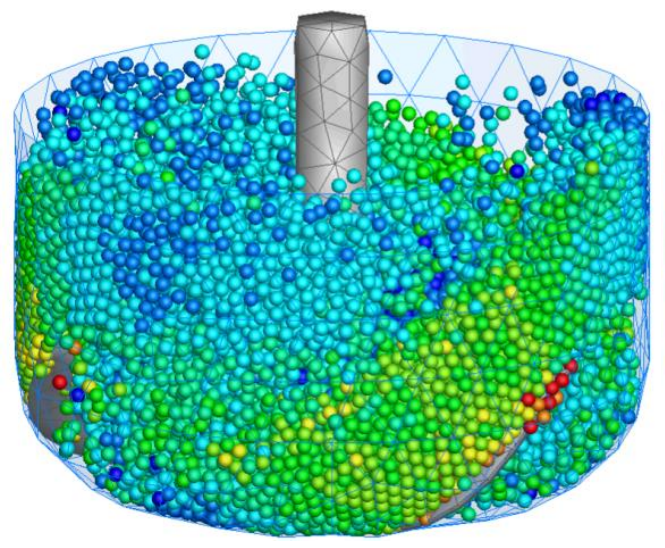

(c)
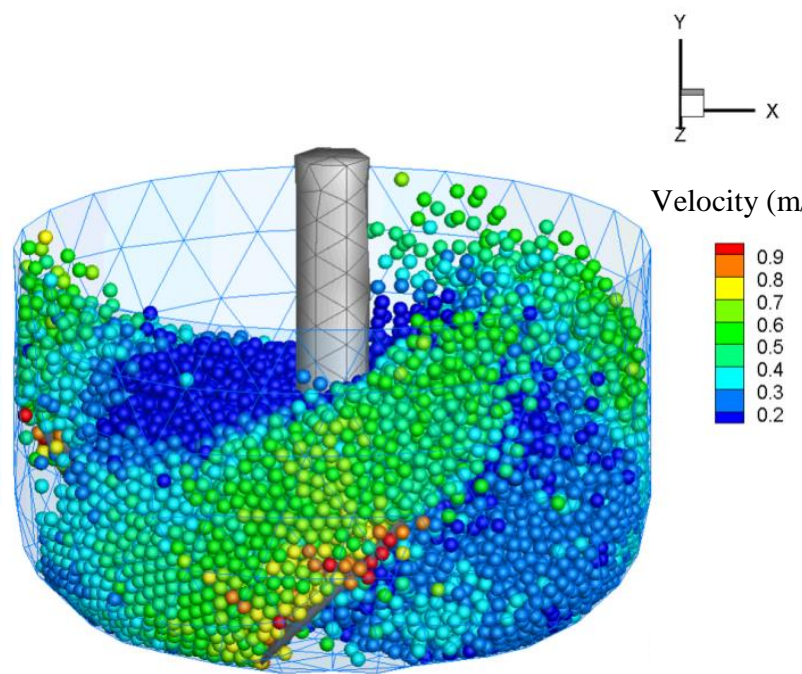

(b)

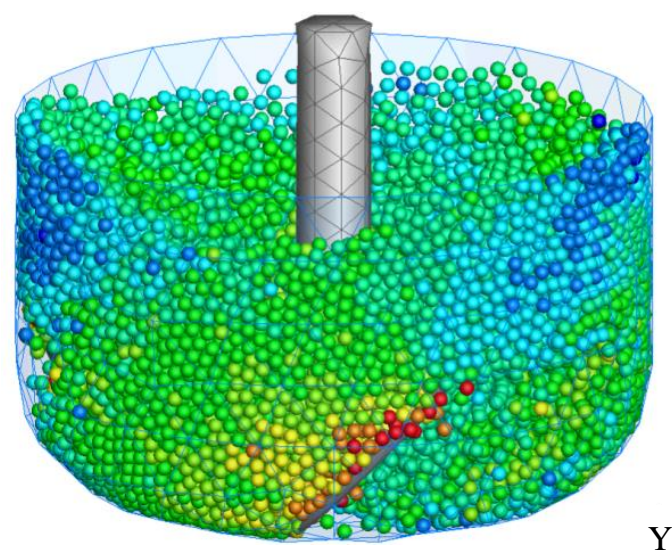

(d)

Figure 8: Particle motion in the Mi-Pro granulator at an impeller speed of $250 \mathrm{RPM}$ at different times after the impeller begins to rotate; a) $0.009 \mathrm{~s}$; b) $0.034 \mathrm{~s}$; c) $0.089 \mathrm{~s} \mathrm{~d}) 0.189 \mathrm{~s}$. The colour of the particle represents the magnitude of its velocity.

\subsection{Particle velocities}

The horizontal blade relative velocity vectors indicate that the major component of the particles velocity is in the tangential direction and slower than the impeller's blade (Figure 9). The velocity vectors in the lower sections of the bowl (Figure 9a) indicate particle motion in the tangential and radial directions, with the largest particle velocities immediately in front of the blade. In this region the particles are moving at the same speed as the impeller and have a slight motion in the radial direction towards the bowl's wall. Behind each of the impeller's blades the particles have a decreased magnitude of velocity. The magnitude continues to decrease until it reaches the next impeller blade. Figures $9 \mathrm{~b}$ and $\mathrm{c}$ depicts the horizontal sections Higher in the bowl. For these sections the majority of the motion is in the tangential direction with the only noticeable motion in the radial direction present where the blades are located and directed towards the bowls centre. 


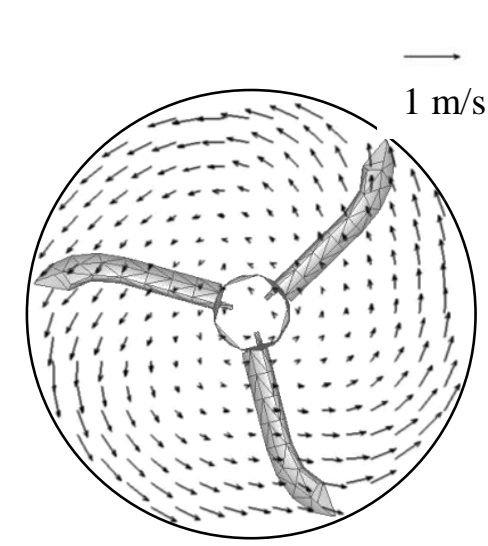

(a) Height $6.75 \mathrm{~mm}$

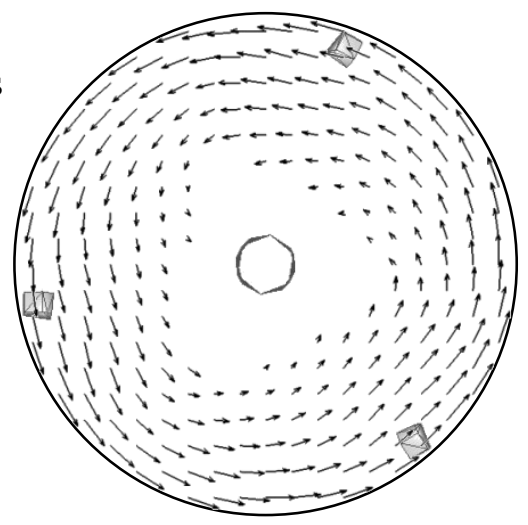

(b) Height $24.75 \mathrm{~mm}$

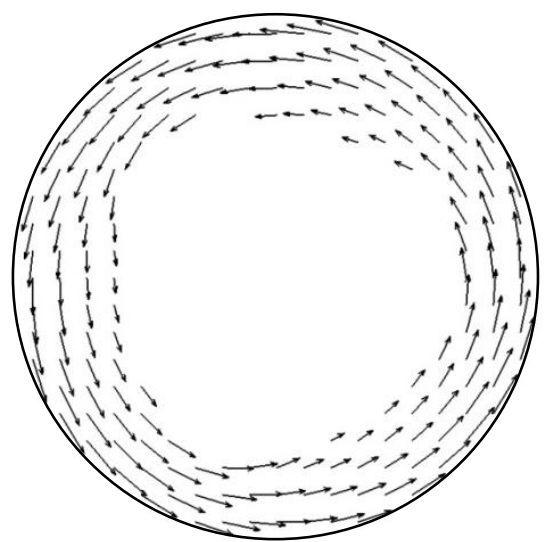

(c) Height $42.75 \mathrm{~mm}$

Figure 9: Blade relative velocity vectors for three different horizontal sections for dry particle flow at an impeller speed of 250 RPM in the Mi-Pro granulator.

Figure 9 highlighted that the largest component of the particles' motion was in the tangential direction. The vertical sections are perpendicular to the tangential direction and only display particle motion in the vertical and radial directions. The impeller's blades are not shown in any of the vertical sections as their complex shape makes it difficult to visualise without over complicating the figure. Figure 10 displays three vertical sections of the Mi-Pro which are located between two of the impeller's blades. The three vertical sections selected were located 0-10 degrees, $40-50$ degrees and 80-90 degrees behind one on the impeller's blades. These represent a vertical section between two impeller blades, one immediately behind an impeller blade, and one at the front of the horizontal part of the impeller blade respectively. In these vertical sections particles are located all the way to the top of the bowl. Particles located in the upper regions are only present towards the side on the bowl's walls. For the three sections presented the particle motion is different. For the sections after the impeller (Figure 10a and $b$ ) the motion is primarily downwards in the vertical section. In the radial direction motion exists and this is recirculating. In the top locations the particles are moving towards the centre of the bowl and in the bottom they are moving towards the bowls walls. For the section before the impeller (Figure 10c) the motion is primarily upwards in the vertical direction. Again the recirculating motion is observed in the radial direction.

Litster et al. (2002) used high speed photography to investigate the powder motion in a lab scale mixer of simple geometry featuring a vertical paddle mixer. Their results showed that two different flow regimes existed depending on impeller speed. At an impeller speed less than 250 RPM bumping would occur, this is where the top surface of the powder moves up and down as the blade agitates the system. At higher impeller speeds a flow they named roping occurs; this is where particles move up the side of the bowl's wall until they reach the top where they fall down and towards the centre of the bowl. This roping motion is similar to that observed from the Mi-Pro simulations. Remy et al. (2010) and Nakamura et al. (2013) created DEM models of four and three bladed impeller mixers respectively and both observed this roping style of motion. 


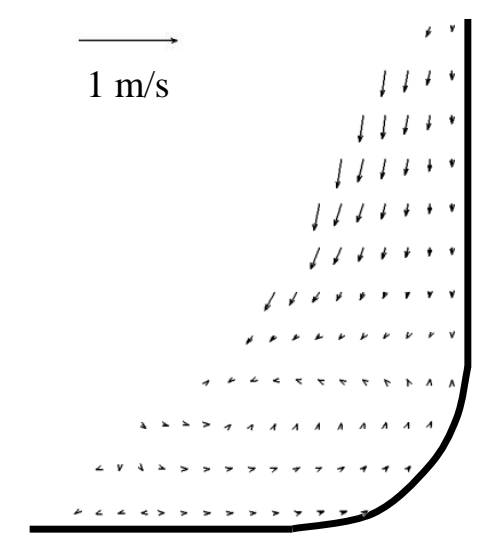

(a) 0-10 degrees

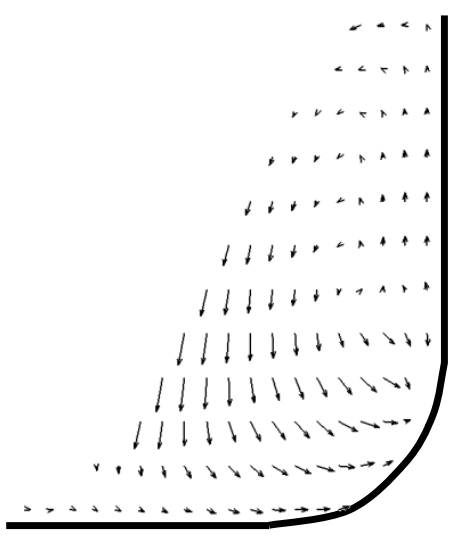

(b) 40-50 degrees $\mathbf{0}^{2}$

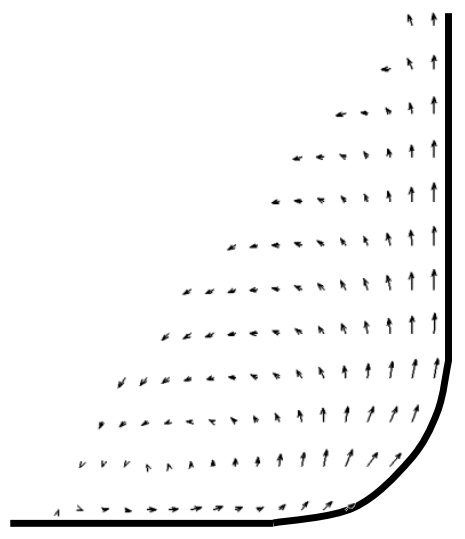

(c) $80-90$ degrees

Figure 10: Particle velocity vectors in different vertical sections for dry particle flow at an impeller speed of 250 RPM in the Mi-Pro granulator.

Figure 11 depicts the average particle tangential velocity at different radial positions and vertical heights. Radial distances with no values for velocity represent a region in the bowl where either no or too few particles were located during the simulation.

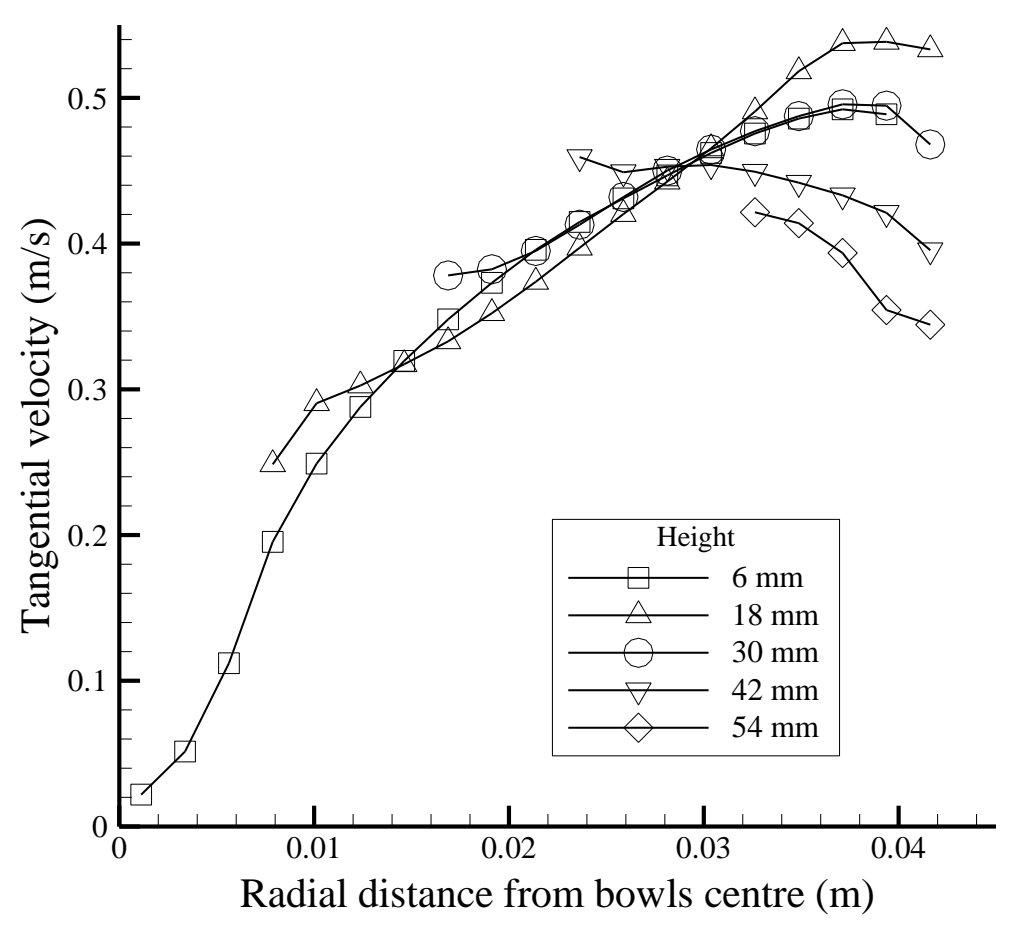

Figure 11: Average tangential velocity at different radial distances from the centre of the bowl at an impeller speed of 250 RPM. These were calculated for different vertical heights.

The tangential velocity profiles had varying features depending upon their height in the bowl. At the heights of 6,18 and $30 \mathrm{~mm}$, the tangential velocities increase with radial distance from the bowl's centre. The maximum average tangential velocity was located just inside the bowl's walls at a height of $18 \mathrm{~mm}$. This coincides with the top of the impeller's blades. Above this point the tangential velocities had reduced magnitude. At heights of 42 and $54 \mathrm{~mm}$ the particles are only located in the 
outer regions of the bowl. At these heights the velocities are greatest towards the centre of the bowl and smallest towards the bowl's walls. All of the tangential velocity profiles, regardless of height, show a reduced velocity when adjacent to the bowl's wall. This is caused by the frictional effect between the particle and the bowl's wall.

\subsection{Packing densities}

Figure 12a depicts the packing density for a bottom section of the bowl. The packing density here is higher than the horizontal sections above it. The packing density in the Mi-Pro reduces with bed height; this is especially noticeable in the top section (Figure 12c) where the particles are only present in the outer region of the bowl. The packing density is less in the upper regions as the particles located here have a free surface towards the bowl's centre. In these higher regions there is no part of the impeller's blade present, therefore, no direct force exists to push the particles together and increase the packing density. For the heights where the impeller's blades are present (Figures 12a and b) a region of low packing density exists behind each blade. This region extends from the impeller's shaft to the bowl's walls where the packing density is at its lowest. Behind the vertical part of the impeller blades few particles are located as most are forced upwards due to being contacted by the impeller's inclined blades.

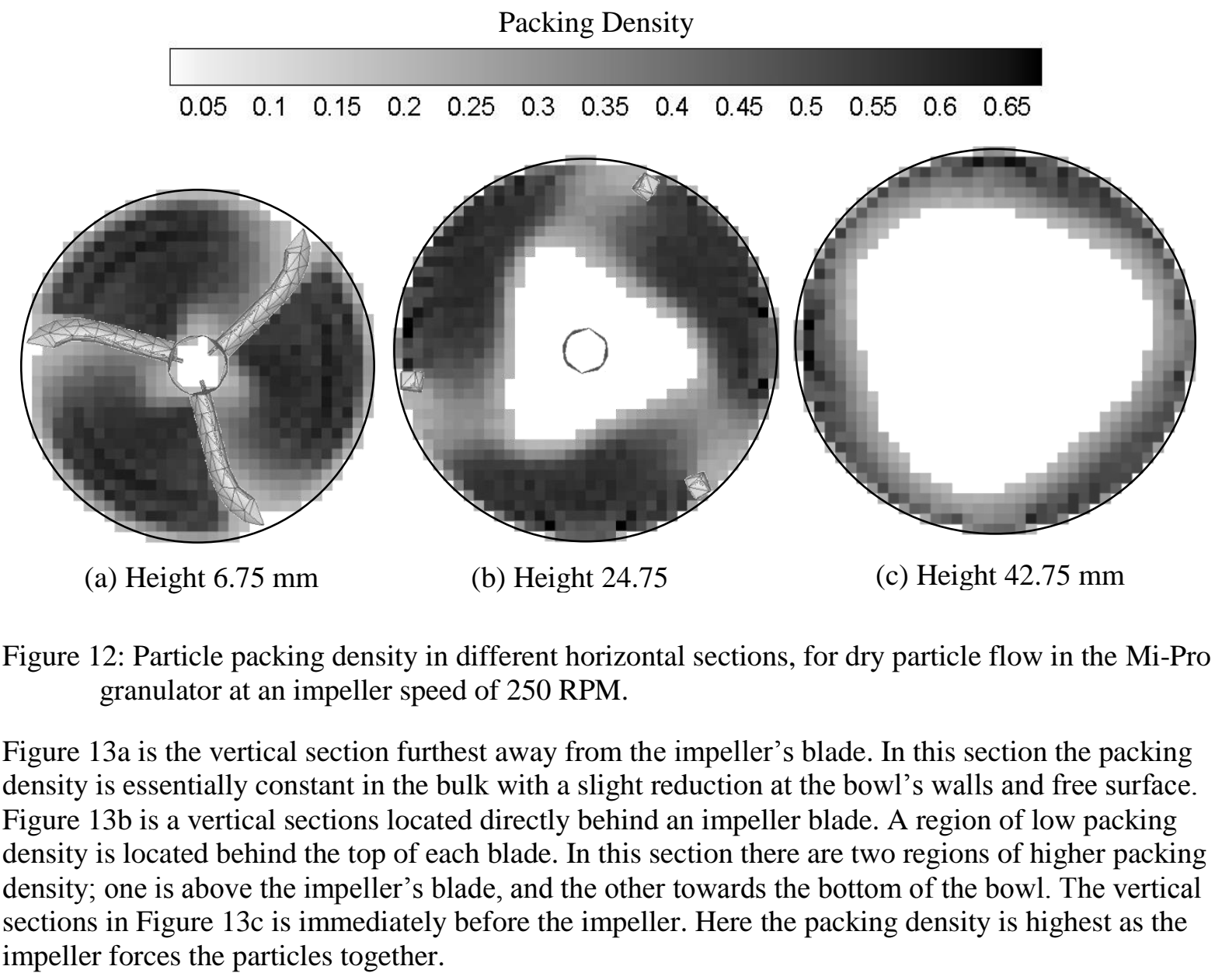




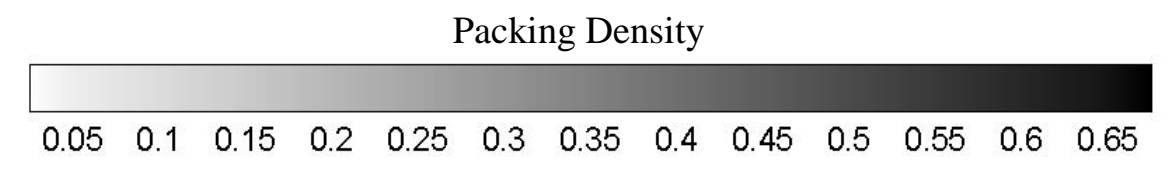

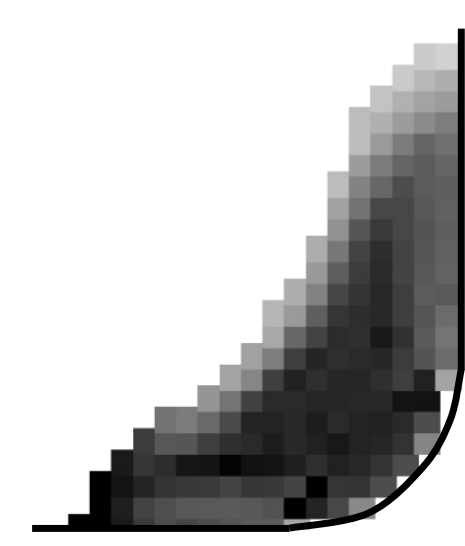

(a) 0-10 degrees

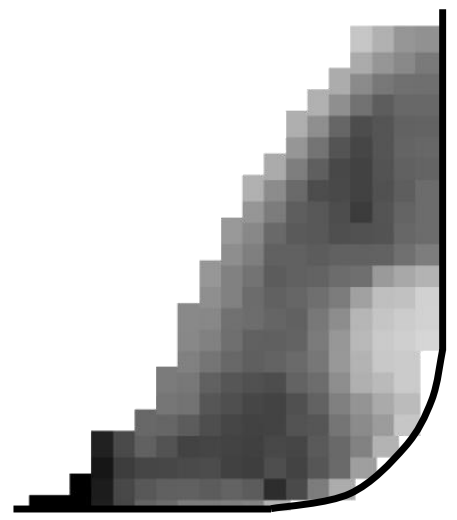

(b) 40-50 degrees

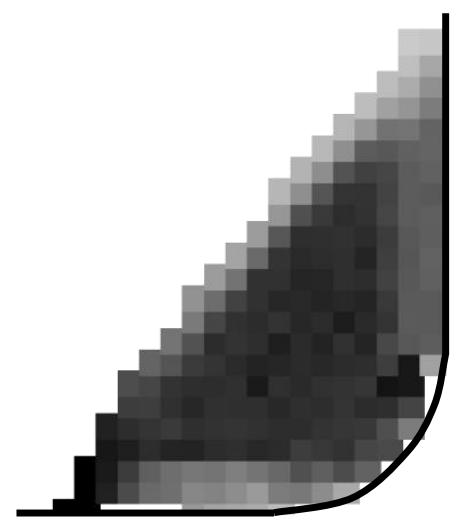

(c) 80-90 degrees

Figure 13: Particle packing density in vertical sections for dry particle flow at $250 \mathrm{RPM}$ in the Mi-Pro granulator.

Sato et al. (2008) created a DEM model of a mixer to investigate the flow patterns and study the torque on the impeller. Their geometry was a simple cylinder fitted with a flat paddle and was not dissimilar to that of Zhou et al. (2004). What made their work different was they used higher impeller speeds (120 to 600 RPM) which were similar to the values used for the Mi-Pro simulations. The size and number of particles were also comparable to the Mi-Pro simulation. At low speeds the flows still formed the heap as already discussed, but at higher speeds this did not happen. Instead the particles were forced upwards by the higher speed of the impeller. In their work they did show the tangential velocities against radial distance from the centre of the bowl. At the impeller speed of 240 RPM, which is the closest to the one used in the Mi-Pro simulation, the tangential velocity profile was very different to the one observed in the Mi-Pro granulator. Their work showed that the particles are only present in the outer two thirds of the bowl and the highest velocities are found nearer the bowl's centre. Nakamura et al. (2009) also used a flat paddle impeller at speeds above 300 RPM and again found that particles moved up the sides of the wall before falling back towards the centre.

Terashita et al. (2002) created a DEM model of a higher shear mixer and used it to determine the optimum fill level which would result in the best granulation conditions. Their work used the concept that particles with the highest average velocities would interact with more particles and this would create an ideal environment for good granulation. The geometry of their granulator was very different to the Mi-Pro; it featured a cylindrical vessel with a flat bottom which became narrower towards the top. The impeller had three blades, each with a uniform cross-section and inclined backwards. In their simulations they had the impeller speed between 120 and 480 RPM. The particle flows in their work were not analysed in a great amount of detail, but they did display particle velocities and particle solid fraction in different sections. Their results showed that the particles were more densely packed towards the sides of the bowl with the highest concentration of particles around the blades. The velocity vectors showed that the highest particle velocities were experienced by particles contacting the impeller blades. These particles would be forced up the side of the bowl's walls before falling back down and towards the centre of the bowl. This is similar to the particle flow observed from the Mi-Pro simulation. In horizontal sections it was shown that the highest velocities were in front of the impeller's blades and behind the blade a region of low velocity and packing density was observed. This low packing density was across the whole area, moved through by the blade which has the same cross section across its length. At the top of the bowl particles were only located against the bowl's walls with reduced velocities. 
So far no existing DEM model has been used to study particle flow within the Mi-Pro. However, Darelius et al. (2007a and 2007b) used experimental methods to study particle flows in the Mi-Pro. The flows were studied using high speed photography and Laser Doppler Anemometry (LDA). LDA is a technique which has the capacity to not only measure surface flow, but also powder flow a small distance into the bed. The Mi-Pro they used was one with a larger size bowl so a direct comparison cannot be made. They used the LDA to study dry mixing in the Mi-Pro and the high speed camera to study wet granulation. For both techniques the flows were recorded at the region next to the bowl's walls, where the tangential and vertical velocities were calculated. For the dry flows it was found that the tangential velocity decreases with an increase in vertical distance from the top of the impeller's blades. This flow feature was also observed from the Mi-Pro simulation. It was found that the particles' velocity increases with small distances away from the bowl's walls; again this was observed from our simulations. When the vertical velocities were recorded it was found that particles were moving upwards at the near wall region, this shows that the roping regime reported from the simulations was also observed experimentally.

For the wet flow the tangential velocity did not decrease with vertical distance from impeller. As the particles became wetter the velocities at the wall increased. This was attributed to the effect of reduced friction between the particles and the bowl's wall. Cavinato et al. (2013) used PIV techniques to measure powder surface velocities in a Mi-Pro fitted with the $1900 \mathrm{ml}$ bowl and an impeller which did not feature the inclined side angles. As the scale and impeller blades were different to the Mi-Pro we used the results were not directly comparable although they were able to image the roping regime at higher impeller speed.

\section{Conclusions}

This article has presented the methodology required to represent a 3D CAD model into a DEM code. This has included how the contact detection is performed and how moving geometry is represented. Suitable selection for the model input parameters is essential to achieve accurate and reliable results. For a DEM model this includes the contact model spring constant and damping coefficient in addition to the simulation time step. Suitable methodologies were presented for all. Before any model can be used it should be validated. This was achieved in our work using high speed photography.

Particle flow behaviour is important for vertical shaft mixers and granulators as they govern the interactions which occur within them and ultimately the effectiveness of the equipment. This is especially the case in granulators where the particle collision dynamics has a large impact on the granulation process. The flow experienced in these types of equipment is complex and often geometry specific. Therefore it is important for industry to have techniques to study the particular piece of equipment they are using. In this work a validated DEM model was developed to investigate dry particle motion in a Mi-Pro lab scale granulator fitted with a $250 \mathrm{ml}$ bowl. This model will be a useful tool which can be used to optimise the geometry of the equipment to give more desirable conditions or to study the effect of particle properties on the complex flow within it. The particle motion in the Mi-Pro is primarily in the tangential direction. This is due to the high speed impeller moving the particles around. As the impeller's blades impact the particles they force them upwards and over them, as well as simultaneously forcing them towards the centre of the bowl. This is an example of the roping flow described by Litster et al. (2002). The highest regions of particle packing density were located in front of each impeller's blades; this results from the blade forcing the particles in front of it together. Behind the vertical section of each blade a wake region of low particle packing density was observed.

\section{Acknowledgements}

The author would like to thank the EPSRC and AstraZeneca for the financial support of this project, and the EPSRC equipment loan pool for the high speed camera used. 


\section{References}

Asmar, B. N., Langston, P. A., Matchett, A. J. \& Walters, J. K. (2002) Validation tests on a distinct element model of vibrating cohesive particle system. Computers and Chemical Engineering, 26, 785-802.

Cavinato, M., Artoni, R., Bresciani, M, Canu, P. \& Santomaso, C. (2013) Scale-up effect on flow patterns in the high shear mixing of cohesive powders. Chemical Engineering Science, 102, $1-9$.

Chandratilleke, G. R., Yu, A. B. \& Bridgwater, J. (2012) A DEM study of the mixing of particles induced by a flat blade. Chemical Engineering Science, 79, 54-74.

Cleary, P. W. (2000) DEM simulation of industrial particle flows: case studies of dragline excavators, mixing in tumblers and centrifugal mills. Powder Technology, 109, 83-104.

Cleary, P. W. \& Hoyer, D. (2000) Centrifugal mill charge motion and power draw: comparison of DEM predictions with experiment. International Journal of Mineral Processing, 59, 131-148.

Cleary, P. W. \& Sawley, M. L (2002) DEM modelling of industrial granular flows: 3D case studies and the effect of particle shape on hopper discharge. Applied Mathematical Modelling, 26, 89-111.

Cundall, P.A. \& Strack, O.D.L. (1979) Discrete numerical-model for granular assemblies. Geotechnique, 29, 47-65.

Darelius, A., Lennartsson, E., Rasmuson, A., Björn, I. N. \& Folestad, S. (2007a) Measurement of the velocity field and frictional properties of wet masses in a high shear mixer. Chemical Engineering Science, 62, 2366-2374.

Darelius, A., Rasmuson, A., Björn, I. N. \& Folestad, S. (2007b) LDA measurements of near wall powder velocities in a high shear mixer. Chemical Engineering Science, 62, 5770-5776.

Di Renzo, A. \& Di Maio, F. P. (2004) Comparison of contact-force models for the simulation of collisions in DEM - based granular flow codes. Chemical Engineering Science, 59, 525-541.

Djordjevic, N., Shi, F. N. \& Morrison, R. (2004) Determination of lifter design, speed and filling effects in AG mills by 3D DEM. Minerals Engineering, 17, 1135-1142.

Gamble, J. F., Dennis, A. B. \& Tobyn, M. (2009) Monitoring and end-point prediction of a small scale wet granulation process using acoustic emission. Pharmaceutical Development and Technology, 14, 3, 299-304.

Hassanpour, A., Pasha, M., Susana, L., Rahmanian, N., Santomaso, A., Ghadiri, M. (2013) Analysis of seeded granulation in high shear granulators by discrete element method. Powder Technology, 238, 50-55.

Hertz, H. (1882) Über die Berührung fester elastischer Körper. Journal fur die reine und angewandte Mathematik, 92, 156-171.

Hoomans, B. P. B., Kuipers, J. A. M. \& Van Swaaij, W. P. M. (2000) Granular dynamics simulation of segregation phenomena in bubbling gas-fluidised beds. Powder Technology, 109, 41-48.

Hua, X., Curtis, J., Hancock, B., Ketterhagen, W. \& Wassgren, C. (2013) The kinematics of noncohesive, sphero-cylindrical particles in a low-speed, vertical axis mixer. Chemical Engineering Science, 101, 144-164.

Iwai, T., Hong, C, W. \& Greil, P. (1999) Fast particle detection algorithms for particle simulations. International Journal of Modern Physics, 10, 5, 823-837.

Kaneko, Y., Shiojima, T. \& Horio, M. (1999) DEM simulation of fluidized beds for gas-phase olefin polymerization. Chemical Engineering Science, 54, 5809-5821.

Kremmer, M. \& Favier, J. F. (2001a) A method for representing boundaries in discrete element modelling, part 1: geometry and contact detection. International Journal for Numerical Methods in Engineering, 51, 12, 1407-1421.

Kremmer, M. \& Favier J. F. (2001b) A method for representing boundaries in discrete element modelling - part 2: Kinematics. International Journal for Numerical Methods in Engineering, 51, 1423-1436.

Kuo, H.P., Knight, P.C., Parker, D.J., Tsuji, Y., Adams, M.J. \& Seville, J.P.K. (2002) The influence of DEM simulation parameters on the particle behaviour in a V-mixer. Chemical Engineering Science, 57, 3621-3638. 
Kuo, H. P., Knight, P. C., Parker, D. J., Adams, M. J. \& Seville, J. P. K. (2004) Discrete element simulation of a high shear mixer. Advanced Powder Technology, 15, 3, 297-309.

Kuwabara, G. \& Kono, K. (1987). Restitution Coefficient in a Collision between 2 Spheres. Japanese Journal of Applied Physics Part 1 - Regular Papers Short Notes \& Review Papers, 26, 1230.

Kwan, C. C., Mio, H., Chen, Y. Q., Ding, Y., Saito, F., Papadopoulos, D. G., Bentham, A. C. \& Ghadiri, M. (2005) Analysis of the milling rate of pharmaceutical powders using the Distinct Element Method (DEM). Chemical Engineering Science, 60, 1441-1448.

Labous, L., Rosato, A.D. \& Dave, R.N. (1997). Measurements of collisional properties of spheres using high-speed video analysis. Physical Review E, 56, 5717.

Langston, P.A. \& Tüzün, U. (1994) Continuous Potential Discrete Particle Simulations of Stress and Velocity-Fields in Hoppers - Transition from Fluid to Granular Flow. Chemical Engineering Science, 49, 1259-1275.

Litster, J. D., Hapgood, K. P., Michaels, J. N., Sims, A., Roberts, M. \& Kameneni S. K. (2002) Scaleup of mixer granulators for effective liquid distribution. Powder Technology, 124, 272-280.

Malone, K. F. \& Xu, B. H. (2008) Determination of contact parameters for discrete element method simulations of granular systems. Particuology, 6, 521-528.

Mikami, T., Kamiya, H. \& Horio, M. (1998) Numerical simulation of cohesive powder behavior in a fluidized bed. Chemical Engineering Science, 53, 1927-1940.

Mishra, B.K. \& Murty, C.V.R. (2001) On the determination of contact parameters for realistic DEM simulations of ball mills. Powder Technology, 115, 290-297.

Mishra, B. K. (2003). A review of computer simulation of tumbling mills by the discrete element method: Part 1 - contact mechanics. International journal of Mineral Processing, 71, 73-93.

Moreno-Atanasio, R. \& Ghadiri, M. (2006) Mechanistic analysis and computer simulation of impact breakage of agglomerates: Effect of surface energy. Chemical Engineering Science, 61, 24762481.

Nakamura, H., Miyazaki, Y., Sato, Y., Iwasaki, T. \& Watano, S. (2009) Numerical analysis of similarities of particle behaviour in high shear mixer granulators with different vessel sizes. Advanced Powder Technology, 20, 5, 493 - 501.

Nakamura, H., Fujii, H. \& Watano, S. (2013) Scale-up of high shear mixer-granulator based on discrete element analysis. Powder Technology, 236, 149-156.

Radl, S., Kalvoda, E., Glasser, B. J. \& Khinast, J. G. (2010) Mixing Characteristics of wet granular matter in a bladed mixer, Powder Technology, 200, 171-189.

Rajamani, R.K., Mishra, B.K., Venugopal, R. \& Datta, A. (2000). Discrete element analysis of tumbling mills. Powder Technology, 109, 105.

Raji, A. O. \& Favier, J. F. (2004) Model for the deformation in agricultural and food particulate materials under bulk compressive loading using discrete element method. 2: Compression of oilseeds. Journal of Food Engineering, 64, 373-380.

Ramos, M.V., Kano, J. \& Kasai, E. (1998). Numerical modeling of granular/particulate systems by discrete element method. High Temperature Materials and Processes, 17, 275.

Remy, B., Canty, T. M., Khinast, J. G. \& Glasser, J. (2010) Experiments and simulations of cohesionless particles with varying roughness in a bladed mixer. Chemical Engineering Science, 65, 4557-4571.

Remy, B., Khinast, J. G. \& Glasser, B. J. (2011) Polydisperse granular flows in a bladed mixer: Experiments and simulations of cohesionless spheres. Chemical Engineering Science, 66, 1811-1824

Rhodes, M.J., Wang, X.S., Nguyen, M., Stewart, P. \& Liffman, K. (2001) Onset of cohesive behaviour in gas fluidized beds: a numerical study using DEM simulation. Chemical Engineering Science, 56, 4433-4438.

Sato, Y., Nakumura, H. \& Watano, S. (2008) Numerical analysis of agitation torque and particle motion in a high shear mixer. Powder Technology, 186, 130-136.

Sinnott, M. \& Cleary, P. (2003) 3D DEM simulations of a high shear mixer. Third International Conference on CFD in the minerals and Process Industries CSIRO, Melbourne, Australia.

Stewart, R. L., Bridgwater., J, Zhou, Y. C. \& Yu, A. B. (2001) Simulated and measured flow of granules in a bladed mixer - a detailed comparison. Chemical Engineering Science, 56, 54575471. 
Tabor, D. (1952) Mechanics of rolling friction I. Phil. Mag. 43, 1055.

Terashita, K., Nishimura, T. \& Natsuyama, S. (2002) Optimization of operating conditions in a highshear mixer using DEM model: Determination of optimal fill level. Chemical and Pharmaceutical Bulletin, 50, 12, 1550-1557.

Thornton, C. (1997) Coefficient of restitution for collinear collisions of elastic perfectly plastic spheres. Journal of Applied Mechanics-Transactions of the Asme, 64, 383-386.

Tijskens, E., Ramon, H. \& De Baerdemaeker, J. (2003) Discrete element modelling for process simulation in agriculture. Journal of Sound and Vibration, 266, 493-514.

Ting, J.M., Corkum, B.T., Kauffman, C.R. \& Greco, C. (1989) Discrete Numerical-Model for Soil Mechanics. Journal of Geotechnical Engineering-Asce, 115, 379-398.

Tsuji, Y., Kawaguchi, T. \& Tanaka, T. (1993) Discrete particle simulation of two-dimensional fluidized bed. Powder Technology, 77, 79-87.

Xu, B.H. \& Yu, A.B. (1997) Numerical simulation of the gas-solid flow in a fluidized bed by combining discrete particle method with computational fluid dynamics. Chemical Engineering Science, 52, 2785-2809.

Xu, B. H., Yu, A. B., Chew, S. J. \& Zulli, P. (1999) A study of the effect of liquid-induced forces on gas-solid flow by a combined continuum and discrete model. Second International Conference on CFD in the Minerals and Process Industries CSIRO, Melbourne, Australia.

Xu, B.H., Yu, A.B. \& Zulli, P. (2001) The effect of the interparticle forces on powder fluidization behaviour. Power and Grains 2001. Sandai, Japan.

Walton, O. R. \& Braun, R. L. (1986) Viscosity, granular-temperature, and stress calculations for shearing assemblies of inelastic, frictional disks. Journal of Rheology, 50, 949-980.

Wang, Y. \& Mason, M.T. (1992). Two-Dimensional Rigid-Body Collisions with Friction. Journal of Applied Mechanics - Transactions of the ASME, 59, 635.

Zhou, Y.C., Wright, B.D., Yang, R.Y., Xu, B.H. \& Yu, A.B. (1999) Rolling friction in the dynamic simulation of sandpile formation. Physica A, 269, 536-553.

Zhou, Y. C., Yu, A. B. \& Bridgwater, J. (2003) Segregation of binary mixture of particles in a bladed mixer. Journal of Chemical Technology and Biotechnology, 78, 187-193.

Zhou, Y. C., Yu, A. B., Stewart, R. L. \& Bridgwater, J. (2004) Microdynamic analysis of the particle flow in a cylindrical bladed mixer. Chemical Engineering Science, 59, 1343-1364. 